\title{
INSTANTONS AND SINGULARITIES IN THE YANG-MILLS FLOW
}

\author{
ALEX WALDRON
}

\begin{abstract}
Several results on existence and convergence of the Yang-Mills flow in dimension four are given. We show that a singularity modeled on an instanton cannot form within finite time. Given low initial self-dual energy, we then study convergence of the flow at infinite time. If an Uhlenbeck limit is anti-self-dual and has vanishing self-dual second cohomology, then no bubbling occurs and the flow converges exponentially. We also recover Taubes's existence theorem, and prove asymptotic stability in the appropriate sense.
\end{abstract}

\section{INTRODUCTION.}

Let $E$ be a vector bundle, with connection $A$, over a Riemannian base manifold $M$. Write $F_{A}$ for the curvature form, $\left|F_{A}\right|^{2}$ for its pointwise norm in a fixed metric, and $D_{A}^{*}$ for the adjoint of the covariant differential. The Yang-Mills flow

$$
\frac{\partial A}{\partial t}=-D_{A}^{*} F_{A}
$$

evolves the connection by the negative gradient of the Yang-Mills functional

$$
\operatorname{YM}(A)=\frac{1}{2} \int_{M}\left|F_{A}\right|^{2} d V .
$$

The Yang-Mills flow first appeared in the work of Atiyah and Bott [1. It was subsequently shown by G. Daskalopoulos [5] over compact manifolds of dimension two, and by Rade [21] in dimensions two and three, that the flow exists for all time and converges. Finite-time blowup is known to occur in dimension five or higher [20, and explicit examples of Type-I shrinking solitons were produced on $\mathbb{R}^{n}, 5 \leq n \leq 9$, by Weinkove [35. Hong and Tian [15] showed that the singular set has codimension at least four, and gave a complex-analytic description in the compact Kahler case, where an application of the maximum principle shows that singularities can form only at infinite time ([27], Ch. 1). In the case of Kahler surfaces, Donaldson's early results [9] for the flow on stable holomorphic bundles have been generalized to unstable bundles by Daskalopoulos and Wentworth ([6], [7]).

The behavior of the flow on general Riemannian manifolds of dimension four, however, has not been understood well. Following the analogy with harmonic map flow in dimension two [28], the foundational work of Struwe [29] gives a global weak solution, not excluding the possibility that point singularities (bubbles) will form within finite time. Previously, outside of the Kahler setting, long-time existence and convergence have only been established by appealing to energy restrictions on blowup limits [23] or by imposing a symmetric Ansatz [25. Moreover, finite-time singularities have long been known as a characteristic feature of critical harmonic map flow [3]. 
This paper provides several new theorems concerning long-time existence (Theorem 2.5, p. 15), smooth convergence (Theorem 3.7, p. 23), and asymptotic stability (Theorems 4.2,4.4, pp. 26-28) of the Yang-Mills flow in dimension four. These results rely on the splitting of two-forms into self-dual and anti-self-dual parts, together with a number of small but useful observations in the parabolic setting. A thorough background section is included (pp. 2 11).

Note on dependence of constants. Our estimates will involve the following constants. C3.2 constant associated to a particular estimate, e.g. Proposition 3.2, where its dependence is stated.

$C$-universal constant; except during the proof, e.g., of Proposition [3.2, where $C=C 3.2$. $C_{S}=1+C_{\mathbb{R}^{4}}$ - Sobolev constant appearing in Section 2.1]

$C_{M}$ - constant depending only on the geometry of $M$.

$C_{A}$-Poincaré constant for a particular connection $A$ obeying (3.12).

$R_{0}$-radius, depending on the geometry of $M$, such that the metric on any geodesic ball of radius less than $R_{0}$ is sufficiently close to Euclidean, in a sense to be determined.

Each of these may increase appropriately, from an earlier to a later appearance; with the exception of $R_{0}$, which may decrease, and $C_{S}$, which is fixed.

\section{Preliminaries}

1.1. Vector bundles and gauge transformations. Let $\pi: E \rightarrow M$ be a vector bundle of rank $n$, with fiberwise inner-product $\langle\cdot, \cdot\rangle$, over a compact, oriented Riemannian base manifold.

A section of $E$ over an open set $U \subset M$ is a smooth map $s: U \rightarrow E$ such that

$$
\pi \circ s=I d_{U} .
$$

By definition, there exists a system of coordinate charts $\left\{U^{a}\right\}$ for $M$, together with a local frame of sections $\left\{e_{\alpha}^{a}\right\}_{\alpha=1}^{n}$ over $U^{a}$ for each $a$, such that any section can be uniquely written

$$
\left.s\right|_{U^{a} \cap U}=\left(s^{a}\right)^{\alpha} e_{\alpha}^{a}
$$

(summing on $\alpha$, not on $a$ ). The functions $\left(s^{a}\right)^{\alpha}$ are referred to as the local components of $s$.

Applying (1.1) with $U=U^{b}$, we may write

$$
\left.e_{\alpha}^{a}\right|_{U^{a} \cap U^{b}}=\left(u^{a b}\right)^{\beta}{ }_{\alpha} e_{\beta}^{b}
$$

in order to define the transition functions $\left(u^{a b}\right)^{\beta}{ }_{\alpha}$. This yields, for any section $s$, the familiar transformation law

$$
\left(s^{b}\right)^{\beta}=\left(u^{a b}\right)_{\alpha}^{\beta}\left(s^{a}\right)^{\alpha} .
$$

By definition, the transition functions (invertible matrices) satisfy the cocycle conditions

$$
u^{b c} \cdot u^{a b}=u^{a c}
$$

on $U^{a} \cap U^{b} \cap U^{c}$. Conversely, these data are sufficient to reconstruct the bundle $E$. 
Choosing the local frames to be orthonormal

$$
\left\langle e_{\alpha}^{a}, e_{\beta}^{a}\right\rangle=\delta_{\alpha \beta}
$$

ensures that the $u^{a b}$ lie inside the orthogonal group $O(n)$. Should these lie within a subgroup $G \subset O(n)$, we say that $E$ has structure group $G$. Since any compact Lie group $G$ embeds into $O(n)$ for some $n$, studying vector rather than principal bundles with compact structure group entails no loss of generality 1

Henceforth, we will suppress the chart label and local frame, writing $s^{\alpha}$ for a section of $E$ in local components, with Greek index, and $s_{\alpha}$ for a section of $E^{*}$. A Latin index $v^{i}$ corresponds to the section $v^{i} \frac{\partial}{\partial x^{i}}$ of the tangent bundle $T M$, and $v_{i}$ to a section $v_{i} d x^{i}$ of the cotangent bundle $T^{*} M$.

The set of gauge transformations $\mathscr{G}_{E} \subset$ End $E$ consists of the orthogonal matrices at each point (or elements of the structure group $G$ ), and a section $u$ of $\left.\mathscr{G}_{E}\right|_{U}$ defines a local metricpreserving automorphism of $E$. The vector bundle of infinitesimal gauge transformations $\mathfrak{g}_{E} \subset$ End $E$ consists of skew-symmetric matrices (or elements of $\mathfrak{g}$ ), and the sections of $\left.\mathfrak{g}_{E}\right|_{U}$ correspond to the Lie algebra of $\left.\mathscr{G}_{E}\right|_{U}$ via exponentiation. We denote the induced action of $u$ on any tensor by $u(\cdot)$, which on $\mathfrak{g}_{E}$ coincides with the adjoint action.

We write $\Omega^{k}(E)$ for the bundle of E-valued $k$-forms, or alternating elements of $\left(T^{*} M\right)^{\otimes k} \otimes$ $E$, with inner-product $\langle\cdot, \cdot\rangle$ induced from the standard orthonormal basis of wedge elements. The components of a two-form $\omega$, for instance, are defined by

$$
\omega=\sum_{i<j} \omega_{i j} d x^{i} \wedge d x^{j}=\frac{1}{2} \omega_{i j} d x^{i} \wedge d x^{j} .
$$

Write $\Omega^{k}\left(\mathfrak{g}_{E}\right) \subset \Omega^{k}($ End $E)$ for the Lie-algebra valued $k$-forms. For $\omega, \eta \in \Omega^{2}($ End $E)$, and similarly for forms of any degree, we define the wedge product

$$
(\omega \wedge \eta)^{\alpha}{ }_{\beta}=\frac{1}{4} \omega_{i j}^{\alpha}{ }_{\gamma} \eta_{k \ell_{\beta}^{\gamma}}\left(d x^{i} \wedge d x^{j} \wedge d x^{k} \wedge d x^{\ell}\right)
$$

Defining the operator $*: \Omega^{k}\left(\mathfrak{g}_{E}\right) \rightarrow \Omega^{4-k}\left(\mathfrak{g}_{E}\right)$ as the linear extension of the ordinary Hodge star on differential forms, we obtain the relation

$$
-\operatorname{Tr} \omega \wedge * \eta=\langle\omega, \eta\rangle d V
$$

for $\omega, \eta \in \Omega^{k}\left(\mathfrak{g}_{E}\right)$.

In dimension four, the Hodge star satisfies

$$
*^{2}=(-1)^{k(4-k)}=(-1)^{k}
$$

on $\Omega^{k}$. For this reason, the two-forms (valued in any bundle) split into orthogonal positive and negative eigenspaces

$$
\Omega^{2}=\Omega^{2+} \oplus \Omega^{2-}
$$

\footnotetext{
${ }^{1}$ It will be clear that if the connection takes values in the Lie algebra $\mathfrak{g}$ of the group $G$, then this property will be preserved as long as we deal with smooth connections and gauge transformations, and in fact more generally (see [10]).
} 
A form $\omega \in \Omega^{2 \pm}$ which satisfies $* \omega= \pm \omega$ is called self-dual or anti-self-dual, respectively. In normal coordinates at a point, (anti)-self-duality amounts to the three relations

$$
\omega_{12}= \pm \omega_{34} \quad \omega_{13}=\mp \omega_{24} \quad \omega_{14}= \pm \omega_{23} .
$$

1.2. Connections and covariant derivatives. A connection $A$ is a metric-preserving rule for transporting fiber elements of $E$, which is linear in the tangent directions of $M$.

Formally, a connection is equivalent to a covariant derivative, or a map

$$
s \mapsto \nabla_{A} s
$$

from sections of $E$ to sections of $T^{*} M \otimes E$, which satisfies

$$
\begin{aligned}
\nabla_{A}(f \cdot s) & =d f \otimes s+f \nabla_{A} s \\
d\langle s, t\rangle & =\left\langle\nabla_{A} s, t\right\rangle+\left\langle s, \nabla_{A} t\right\rangle
\end{aligned}
$$

for any smooth function $f$. In local coordinates, writing $\left(\nabla_{A} s\right)\left(\partial_{i}\right)=\nabla_{i} s$, we may define the connection components

$$
A_{i \beta}^{\alpha}=\left\langle e_{\alpha}, \nabla_{i} e_{\beta}\right\rangle
$$

in order to obtain the well-known formula

$$
\nabla_{i} s^{\alpha}:=\left(\nabla_{A} s\right)_{i}{ }^{\alpha}=\partial_{i} s^{\alpha}+A_{i \beta}^{\alpha} s^{\beta} .
$$

Under a gauge transformation or change-of-frame $u$, the components of $A$ must transform according to the requirement

$$
u\left(\nabla_{A} s\right)=\nabla_{u(A)}(u(s))
$$

or in matrix notation

$$
u(A)=u \cdot A \cdot u^{-1}-d u \cdot u^{-1} .
$$

From this transformation law, it is evident that the difference of any two connections defines a genuine section of $\Omega^{1}\left(\mathfrak{g}_{E}\right)$, as does the derivative $\dot{A}$ of a smooth family of connections. The set of all connections is thus an affine space $\mathscr{A}_{E}$ modeled on $\Omega^{1}\left(\mathfrak{g}_{E}\right)$.

Define the covariant differential on sections $\Omega^{k}(E) \rightarrow \Omega^{k+1}(E)$ by the rule

$$
D_{A}\left(s^{\alpha} d x^{i_{1}} \wedge \cdots \wedge d x^{i_{k}}\right)=\nabla_{i} s^{\alpha} d x^{i} \wedge d x^{i_{1}} \wedge \cdots \wedge d x^{i_{k}} .
$$

By abuse of notation, we may consider $A$ in local coordinates as a $\mathfrak{g}$-valued "connection 1-form," $A_{i \beta}^{\alpha} d x^{i}$, and rewrite $D_{A}$ in terms of the wedge product, as follows. For $\alpha \in \Omega^{k}(E)$, we will write

$$
D_{A} \alpha=d \alpha+A \wedge \alpha
$$

and for $\omega \in \Omega^{k}(\operatorname{End} E)$

$$
D_{A} \omega=d \omega+A \wedge \omega+(-1)^{k+1} \omega \wedge A .
$$

We will choose based on efficiency whether to employ the form or the index notation in each derivation that follows. The adjoint of the covariant derivative is given by

$$
\left(\nabla_{A}^{*} \omega\right)_{i_{1} \cdots i_{k}}=-g^{\ell j} \nabla_{\ell} \omega_{j i_{1} \cdots i_{k}}=-\nabla^{j} \omega_{j i_{1} \cdots i_{k}}
$$


which agrees, on form components, with the adjoint of the covariant differential

$$
D_{A}^{*}=-* D_{A} *
$$

1.3. Curvature and Bianchi identities. The curvature $F_{A}$ of the connection $A$ is defined as the operator on sections of $E$

$$
\begin{aligned}
\left(D_{A}\right)^{2} s & =D_{A}(d s+A \cdot s) \\
& =d^{2} s+d A \cdot s-A \wedge d s+A \wedge d s+A \wedge A \cdot s \\
& =(d A+A \wedge A) s .
\end{aligned}
$$

This operator is evidently $C^{\infty}$-linear, and defines a section

$$
\frac{1}{2} F_{i j} d x^{i} \wedge d x^{j} \in \Omega^{2}\left(\mathfrak{g}_{E}\right)
$$

with components

$$
F_{i j}{ }^{\alpha}{ }_{\beta}=\partial_{i} A_{j \beta}^{\alpha}-\partial_{j} A_{i \beta}^{\alpha}+A_{i \gamma}^{\alpha} A_{j \beta}^{\gamma}-A_{j \gamma}^{\alpha} A_{i \beta}^{\gamma} .
$$

Writing $R_{i j}^{k} \ell$ for the curvature of $\Gamma$ on $T M$, we obtain the commutation formula

$$
\begin{aligned}
{\left[\nabla_{i}, \nabla_{j}\right] t^{k} \ell_{\beta}^{\alpha}=} & R_{i j}{ }^{k}{ }_{m} t^{m} \ell^{\alpha}{ }_{\beta}-R_{i j}{ }^{n} \ell t^{k}{ }_{n}{ }_{\beta}{ }_{\beta} \\
& +F_{i j}{ }^{\alpha}{ }_{\gamma} t^{k}{ }_{j}{ }_{\beta}-F_{i j}{ }^{\gamma}{ }_{\beta} t^{k}{ }_{j}{ }^{\alpha} \gamma
\end{aligned}
$$

and similar formulae in general. Note the identity

$$
\begin{aligned}
\left(D_{A}^{*}\right)^{2} F_{A} & =\nabla^{i} \nabla^{j} F_{i j}=\frac{1}{2}\left(\nabla^{i} \nabla^{j}-\nabla^{j} \nabla^{i}\right) F_{i j} \\
& =\frac{1}{2}\left(-R^{i j n}{ }_{i} F_{n j}-R_{j}^{i j n} F_{i n}+\left[F^{i j}, F_{i j}\right]\right) \\
& =0 .
\end{aligned}
$$

We have also the second Bianchi identity

$$
\begin{aligned}
D_{A} F_{A} & =d(d A+A \wedge A)+A \wedge d A-d A \wedge A+A \wedge(A \wedge A)-(A \wedge A) \wedge A \\
& =d^{2} A+d A \wedge A-A \wedge d A+A \wedge d A-d A \wedge A \\
& =0 .
\end{aligned}
$$

The latter is equivalent to the familiar identity on component matrices

$$
\nabla_{i} F_{j k}+\nabla_{j} F_{k i}+\nabla_{k} F_{i j}=0
$$

1.4. Yang-Mills and instantons. The $L^{2}$ gradient of the Yang-Mills energy is obtained as follows.

For a one-form $a \in \Omega^{1}\left(\mathfrak{g}_{E}\right)$, note the formula

$$
\begin{aligned}
F_{A+a} & =F_{A}+d a+A \wedge a+a \wedge A+a \wedge a \\
& =F_{A}+D_{A} a+a \wedge a
\end{aligned}
$$


and compute

$$
\begin{aligned}
\left.\frac{d}{d t} Y M(A+t a)\right|_{t=0} & =\frac{1}{2} \frac{d}{d t}\left(\int\left(\left|F_{A}\right|^{2}+2 t\left\langle F_{A}, D_{A} a\right\rangle\right) d V+O\left(t^{2}\right)\right) \\
& =\int\left\langle a, D_{A}^{*} F_{A}\right\rangle d V .
\end{aligned}
$$

The Yang-Mills flow is therefore defined as above, or in local components

$$
\frac{\partial}{\partial t} A_{j \beta}^{\alpha}=\nabla^{i} F_{i j}^{\alpha}{ }^{\alpha}
$$

By construction, any sufficiently smooth solution will satisfy the energy inequality

$$
\mathrm{YM}(A(0))-\mathrm{YM}(A(T))=\int_{0}^{T}\left\|D_{A}^{*} F_{A}\right\|^{2} d t
$$

We may therefore expect a weak limit

$$
A(t) \rightarrow A_{\infty} \quad(t \rightarrow \infty)
$$

which, if not a minimum of YM, is at least a Yang-Mills connection, satisfying

$$
D_{A_{\infty}}^{*} F_{A_{\infty}}=0 \text {. }
$$

Note that we will often abbreviate

$$
\|\cdot\|=\|\cdot\|_{L^{2}(M)} \cdot
$$

For the self-dual and anti-self-dual parts of the curvature form, write

$$
F^{ \pm}=\frac{1}{2}(F \pm * F)
$$

respectively. From the second Bianchi identity, remark that

$$
\begin{aligned}
2 D^{*} F^{ \pm} & =-*\left(D * F \pm D *^{2} F\right) \\
& =D^{*} F .
\end{aligned}
$$

Therefore, if a connection is self-dual $\left(F^{-}=0\right)$ or anti-self-dual $\left(F^{+}=0\right)$, it is a critical point of the Yang-Mills energy. These special Yang-Mills connections are called instantons.

Recall from Chern-Weil theory that the characteristic number

$$
\kappa(E)=\frac{1}{8 \pi^{2}} \int \operatorname{Tr} F \wedge F
$$

is a topological invariant of $E$, which for complex bundles coincides with the second Chern character. From (1.3), we compute

$$
\begin{aligned}
\int \operatorname{Tr} F \wedge F & =-\int\left\langle F^{+}+F^{-}, F^{+}-F^{-}\right\rangle d V \\
& =\left\|F^{-}\right\|^{2}-\left\|F^{+}\right\|^{2}
\end{aligned}
$$

but by orthogonality, also

$$
\|F\|^{2}=\left\|F^{+}\right\|^{2}+\left\|F^{-}\right\|^{2}
$$

We obtain the formula

$$
\|F\|^{2}=8 \pi^{2} \kappa+2\left\|F^{+}\right\|^{2}
$$


A connection is therefore anti-self-dual if and only if it attains the minimum energy $4 \pi^{2} \kappa$.

Assuming $\kappa(E) \geq 0$, without loss of generality, one might expect to find anti-self-dual instantons on $E$ - a principal aim in Donaldson theory ([8], [10]).

1.5. Evolution of curvature and Weitzenbock formulae. From (1.10), we compute the evolution

$$
\frac{\partial}{\partial t} F_{A}=D_{A}\left(-D_{A}^{*} F_{A}\right)
$$

In view of the second Bianchi identity, we may rewrite this as the tensorial heat equation

$$
\left(\frac{\partial}{\partial t}+\Delta_{A}\right) F_{A}=0
$$

where $\Delta_{A}=D_{A} D_{A}^{*}+D_{A}^{*} D_{A}$ is the Hodge Laplacian with respect to the evolving connection $A=A(t)$. Henceforth we suppress the label and write $D=D_{A}, \nabla=\nabla_{A}$, etc., although for emphasis we continue to denote the Hodge Laplacian by $\Delta_{A}$.

We compute, for $\omega \in \Omega^{k}\left(\mathfrak{g}_{E}\right)$

$$
\begin{aligned}
\left(D^{*} D+D D^{*}\right) \omega_{i_{1} \cdots i_{k}} & =-\nabla^{j}\left(\nabla_{j} \omega_{i_{1} \cdots i_{k}}-\nabla_{i_{1}} \omega_{j i_{2} \cdots i_{k}}-\cdots-\nabla_{i_{k}} \omega_{i_{1} \cdots i_{k-1} j}\right) \\
& -\nabla_{i_{1}} \nabla^{j} \omega_{j i_{2} \cdots i_{k}}+\nabla_{i_{2}} \nabla^{j} \omega_{j i_{1} i_{3} \cdots i_{k}}+\cdots+\nabla_{i_{k}} \nabla^{j} \omega_{j i_{2} \cdots i_{k-1} i_{1}} .
\end{aligned}
$$

Permuting $j$ and $i_{1}$ in the + terms of the second line, we may group all but the first term of the first line into commutators. We obtain the Weitzenbock formula

$$
\left(\Delta_{A} \omega\right)_{i_{1} \cdots i_{k}}=\nabla^{*} \nabla \omega_{i_{1} \cdots i_{k}}-\left[F_{i_{1}}{ }^{j}, \omega_{j i_{2} \cdots i_{k}}\right]-\cdots-\left[F_{i_{k}}{ }^{j}, \omega_{i_{1} \cdots i_{k-1} j}\right]+R m \# \omega
$$

where \# is a bilinear expression in the tensor components. In particular, for a two-form, we have

$$
\begin{aligned}
-\left(\Delta_{A} \omega\right)_{i j} & =\nabla^{k} \nabla_{k} \omega_{i j}+\left[F_{i}{ }^{k}, \omega_{k j}\right]-\left[F_{j}{ }^{k}, \omega_{k i}\right] \\
& -R_{i}{ }^{k \ell}{ }_{k} \omega_{\ell j}-R_{i}{ }^{k \ell}{ }_{j} \omega_{k \ell}+R_{j}{ }^{k \ell}{ }_{k} \omega_{\ell i}+R_{j}{ }^{k \ell}{ }_{i} \omega_{k \ell} .
\end{aligned}
$$

We now make a simple observation about the zeroth-order terms - see [17], Appendix II. For $\omega \in \Omega^{2+}$ and $\eta \in \Omega^{2-}$, we may write in normal coordinates

$$
\begin{aligned}
\omega_{1 k} \eta_{k 2}-\omega_{2 k} \eta_{k 1} & =\omega_{13} \eta_{32}-\omega_{23} \eta_{31}+\omega_{14} \eta_{42}-\omega_{24} \eta_{41} \\
& =\left(-\omega_{24}\right)\left(-\eta_{41}\right)-\omega_{14} \eta_{42}+\omega_{14} \eta_{42}-\omega_{24} \eta_{41} \\
& =0
\end{aligned}
$$

and likewise for any choice of indices. A similar calculation shows that for $\omega, \bar{\omega}$ self-dual, $\omega_{i k} \bar{\omega}_{k j}-\omega_{j k} \bar{\omega}_{k i}$ is again self-dual. These facts amount to the well-known splitting of Lie algebras

$$
s o(4)=s o(3) \oplus s o(3) \text {. }
$$

For the $R m$ terms of (1.13), one notes that the first and third are skew in $i, j$, as are the second and fourth, and that these are each self-dual if the same is true of $\omega .2$

\footnotetext{
${ }^{2}$ See Freed and Uhlenbeck [1], Appendix C, for an explanation of the splitting, and for the derivation of the well-known expression " $R m \#=W^{+}-R / 3$ " in (1.14).
} 
We conclude that the extra terms of the Weitzenbock formula (1.13) in fact split into selfdual and anti-self-dual parts. Note also that $\Delta_{A} *=* \Delta_{A}$, and the trace Laplacian preserves self-duality since the same is true of the (metric-compatible) covariant derivative. Hence

$$
-\left(\Delta_{A} \omega\right)_{i j}=\nabla^{k} \nabla_{k} \omega_{i j}+\left[F_{i}^{+k}, \omega_{k j}\right]-\left[F_{j}^{+k}, \omega_{k i}\right]+R m \# \omega
$$

for $\omega$ self-dual, and a similar formula holds for anti-self-dual forms. Applied to the self-dual curvature $F^{+}$, (1.14) yields the key evolution equation

$$
\frac{\partial}{\partial t} F_{i j}^{+}=\nabla^{k} \nabla_{k} F_{i j}^{+}+2\left[F_{i}^{+k}, F_{k j}^{+}\right]+R m \# F^{+}
$$

1.6. Sobolev spaces. Fix, for the remainder of the paper, a smooth reference connection $\nabla_{\text {ref }}$ on $E$. For $\omega \in \Omega^{k}($ End $E)$ and any domain $\Omega \subset M$, define the Sobolev norms

$$
\|\omega\|_{L_{k}^{p}(\Omega)}=\left(\sum_{\ell=0}^{k}\left\|\nabla_{r e f}^{(\ell)} \omega\right\|_{L^{p}(\Omega)}^{p}\right)^{1 / p}
$$

and also write

$$
H^{k}(\Omega)=L_{k}^{2}(\Omega)
$$

By (1.5), any connection can be uniquely written $D_{r e f}+A$, with $A \in \Omega^{1}\left(\mathfrak{g}_{E}\right)$. We may therefore define the Sobolev norm of a connection, $\|A\|_{L_{k}^{p}(\Omega)}$, as the norm of this corresponding one-form 3 The Sobolev space of $L_{k}^{p}(\Omega)$ connections is defined as the completion of $\left.\mathscr{A}_{E}\right|_{\Omega}$ with respect to the $L_{k}^{p}(\Omega)$ norm. All connections appearing in this paper will be assumed to be smooth, unless explicitly stated otherwise.

For two open sets $\Omega_{1} \subset \subset \Omega \subset M$, there is a local Sobolev inequality

$$
\|\omega\|_{L^{4}\left(\Omega_{1}\right)} \leq C_{[1.16}\|\omega\|_{H^{1}(\Omega)} .
$$

This follows from the ordinary Sobolev inequality in dimension four and the Kato inequality; hence $C_{1.16}$ depends on $\Omega_{1}$ and $\Omega$, but not $\nabla_{\text {ref }}$. Henceforth, unless otherwise stated, we will also abbreviate $L_{k}^{p}=L_{k}^{p}(M)$.

From (1.16), note that the Yang-Mills energy is controlled locally by the $H^{1}$ norm of the connection. The converse also holds true for connections of small energy, after choosing a local Coulomb gauge [32. However, due to the zeroth-order terms of the Weitzenbock formula, the Sobolev constant for $D_{A} \oplus D_{A}^{*}$ may blow up as the curvature of $A$ concentrates.

1.7. Short-time existence. For the benefit of the reader, we briefly recall the construction of a solution to the Yang-Mills flow

$$
D(t)=D_{\text {ref }}+A(t)
$$

on a maximal time interval $0 \leq t<T$, due in dimension four independently to Struwe [29] and to Kozono, Maeda, and Naito [16]. In subsequent sections, $A(t)$ will always denote a solution of the form described here, which will in particular be smooth for $0<t<T$ (after a fixed

\footnotetext{
${ }^{3} \mathrm{~A}$ different choice of reference connection yields uniformly equivalent norms. It is important to emphasize, however, that the zeroth-order norms of a difference of two connections, being a genuine 1-form, does not depend on the choice of gauge (or on $\nabla_{r e f}$ ), and moreover that $\mathscr{G}_{E}$ acts by isometries for the $L^{p}=L_{0}^{p}$ distances on the affine space $\mathscr{A}_{E}$.
} 
change of gauge). Because the main interest of this paper is not short-time existence theory, but rather long-time existence and convergence, the discussion will be slightly informal at times.

1.7.1. Smooth initial data. For a sufficiently smooth initial connection, short-time existence follows by a De Turck-type trick first employed by Donaldson [9] (see also [10], §6).

Assume first that $D(t)$ is a family of connections depending smoothly on time, $u_{t} \in \mathscr{G}_{E}$ is a family of gauge transformations with $u_{t_{0}}=1$, and define

$$
\bar{D}(t)=u_{t}(D(t))
$$

The transformation law (1.5) and the Leibniz rule give

$$
\left.\frac{d}{d t} \bar{D}\right|_{t=t_{0}}=\left.\frac{d}{d t} D\right|_{t=t_{0}}-D s
$$

where $s=\left.\frac{d}{d t} u_{t}\right|_{t=t_{0}}$. In general, consider the gauge transformation $u_{t} \cdot u_{t_{0}}^{-1}$ in (1.17), and apply $u_{t_{0}}$ to both sides. Letting

$$
s(t)=u_{t}^{-1} \frac{d}{d t} u_{t} \in \mathfrak{g}_{E}
$$

this yields

$$
\left.\frac{d}{d t} \bar{D}\right|_{t=t_{0}}=u_{t_{0}}\left(\left.\frac{d}{d t} D\right|_{t=t_{0}}\right)-\bar{D} s\left(t_{0}\right) .
$$

Now fix a smooth connection $D_{1}$. To solve the Yang-Mills flow for a smooth initial connection $D_{0}=D_{1}+A_{0}$, write $\bar{D}(t)=D_{1}+a(t)$ and consider the alternate equation

$$
\frac{d}{d t} \bar{D}=\frac{d}{d t} a=-\bar{D}^{*} \bar{F}+\bar{D}\left(-\bar{D}^{*} a\right), \quad a(0)=A_{0}
$$

Recall that

$$
\bar{F}=F_{D_{1}}+D_{1} a+a \wedge a=F_{D_{1}}+\bar{D} a+a \# a
$$

so we may rewrite (1.19)

$$
\begin{aligned}
\frac{d}{d t} a & =-\left(\bar{D} \bar{D}^{*}+\bar{D}^{*} \bar{D}\right) a-\bar{D}^{*}\left(F_{D_{1}}+a \# a\right) \\
& =-\Delta_{D_{1}} a+P\left(a, \nabla_{1} a\right) .
\end{aligned}
$$

with $P$ a polynomial function of the tensor components with smooth coefficients.

The Sobolev multiplication theorems - see [11], Appendix A-imply that for $k \geq k_{0}$ sufficiently large, $P$ determines a smooth map of Hilbert spaces

$$
H^{k}\left(\Omega^{1}\left(\mathfrak{g}_{E}\right)\right) \rightarrow H^{k-1}\left(\Omega^{1}\left(\mathfrak{g}_{E}\right)\right) .
$$

Standard parabolic theory - in particular Lemma 3.2 of Struwe [29], and a straightforward instance of the fixed-point argument used in proving Theorem 1.1 below-provides a unique solution $a(t)$ for $0 \leq t \leq \tau$, with $\tau$ depending only on $D_{1}$ and a fixed upper bound for $\left\|A_{0}\right\|_{H^{k}}$. 
This solution is continuous in $H^{k}$ with respect to time and initial data-see Lions-Magenes [19] for basic theory 4

Since $D_{0}$ is smooth and $k \geq k_{0}$ was arbitrary, the solution $\bar{D}(t)$ is smooth. We may therefore define a smooth gauge-transformation $u=u_{t}$ by the pointwise ODE

$$
s=u^{-1} \frac{d}{d t} u=\bar{D}^{*} a, \quad u_{0}=1 .
$$

By (1.19), $D(t)=u^{-1}(\bar{D})$ is a classical solution of the Yang-Mills flow.

This solution can then be extended in the usual way: provided that there exists a smooth limit $D(t) \rightarrow D(T)$ as $t \rightarrow T$ over all of $M$, we may continue the flow by concatenating another short-time solution with initial data $D(T)$. Although the intrinsic characterization of the maximal smooth extension time $T$ follows directly from the parabolic estimates of Section 3.1 below, it will be useful to recall Struwe's sharp local existence result and to summarize his method.

1.7.2. Rough initial data-Struwe's approach. For initial data $D_{0} \in H^{1}$, Struwe [29] writes

$$
\bar{D}(t)=D_{1}+\bar{A}(t)=D_{1}+A_{b g}(t)+a(t)
$$

where $D_{1}$ is a smooth connection near $D_{0}$, and $A_{b g}$ solves the ordinary heat equation with respect to $D_{1}$, with

$$
A_{b g}(0)=A_{0}=D_{0}-D_{1} .
$$

The remaining piece $a(t)$, with $a(0)=0$, is determined by a fixed-point argument based on sharper estimates for the nonlinearity $P$ defined above.

Theorem 1.1. (Struwe [29], §4.2-4.3) Given a smooth connection $D_{1}$, there exist $Q_{1.1}$ and $\epsilon>0$ (depending only on $\left(E, D_{\text {ref }}\right)$ ) and $\tau$ (depending on $D_{1}$ ) as follows. For any $A_{0} \in H^{1}$ with $\left\|A_{0}\right\|_{H^{1}}<\epsilon$, there exists a smooth solution $\bar{D}(t)=D_{1}+\bar{A}(t)$ to (1.19) for $0<t \leq \tau$, with

$$
\|\bar{A}(t)\|_{H^{1}} \leq Q_{1.1}\left\|A_{0}\right\|_{H^{1}}
$$

and $\bar{A}(t) \rightarrow A_{0}$ strongly in $H^{1}$ as $t \rightarrow 0$.

The desired weak solution $D(t)$ of the Yang-Mills flow, in the sense defined by Struwe, is then obtained as follows. Fix a time $0<t_{0}<\tau$, and let $\hat{D}(t)$ be the solution with

$$
\hat{D}\left(t_{0}\right)=\bar{D}\left(t_{0}\right)
$$

obtained by solving (1.21) for $0<t<\tau$. For any sequence of times $t_{i} \rightarrow 0$, by construction there exist smooth gauge transformations $u_{i}$ such that

$$
u_{i}\left(\hat{D}\left(t_{i}\right)\right)=\bar{D}\left(t_{i}\right) .
$$

Theorem 1.1 implies

$$
u_{i}\left(\hat{D}\left(t_{i}\right)\right) \stackrel{H^{1}}{\longrightarrow} D_{0} .
$$

\footnotetext{
${ }^{4}$ See also Rade 21] for a slightly different method, giving the optimal $k_{0}$ for which these properties hold.

${ }^{5}$ Throughout the paper, a smooth limit will mean a limit in $C^{\infty}$, i.e. $C^{k}$ for all $k$, over the domain $\Omega \subset M$ in question.
} 
Struwe also finds a strong $H^{1}$ limit $u_{i} \rightarrow u_{0}$, and defines $D(t)=u_{0}(\hat{D})$ as a weak solution with

$$
D(t) \stackrel{L^{2}}{\longrightarrow} D_{0}
$$

as $t \rightarrow 0$. The solution $D(t)$ is smooth for $0<t<\tau$, modulo the constant gauge transformation $u_{0}$.

1.7.3. Energy concentration. Both the constructions of Struwe, and of Kozono et. al. [16], yield the following criterion for long-time existence. For a certain $\epsilon_{0}>0$, we say that the curvature $F(t)=F_{A(t)}$ concentrates (in $L^{2}$ ) at $x \in M$ if

$$
\inf _{R>0} \limsup _{t \rightarrow T} \int_{B_{R}(x)}|F(t)|^{2} d V \geq \epsilon_{0} .
$$

Theorem 1.2. The maximal smooth existence time $T$, is characterized by concentration of the curvature $F(t)$ at some $x \in M$ as $t \rightarrow T$.

\section{Finite-time existence}

In view of Theorem 1.2, to prove long-time existence of the Yang-Mills flow in dimension four, it remains to control the concentration of curvature.

The first step is to obtain separate control of the self-dual curvature directly from (1.15). Applying the inner-product with $F^{+}$yields

$$
\left(\frac{\partial}{\partial t}+\Delta\right)\left|F^{+}\right|^{2}=-2\left|\nabla F^{+}\right|^{2}+2\left\langle F^{+i j},\left[F_{i}^{+k}, F_{k j}^{+}\right]\right\rangle+R m \# F^{+} \# F^{+} .
$$

Writing $u=\left|F^{+}\right|^{2}$, we obtain the differential inequality

$$
\left(\frac{\partial}{\partial t}+\Delta\right) u \leq A u^{3 / 2}+B u
$$

where $A$ is a universal constant, and $B$ is a universal constant times $\|R m\|_{L^{\infty}(M)}$. Similar inequalities hold for the anti-self-dual curvature $F^{-}$, and the full curvature $F$.

2.1. Estimates on curvature evolution. Estimates for (2.1) follow by adapting Moser iteration to the present borderline situation. This can be done in two different ways. Apart from Theorem 4.2, later results will only require Proposition 2.3, with $p=1$.

Proposition 2.1. Let $u$ be a nonnegative smooth function satisfying (2.1) on $M \times[0, T)$, with $M$ compact of dimension four. There exists a universal constant $\delta>0$, and $R_{0}>0$, depending on the geometry of $M$, as follows. If $R<R_{0}$ is such that

$$
\int_{B_{R}\left(x_{0}\right)} u(t)<\delta^{2}
$$

\footnotetext{
${ }^{6}$ Although the short-time solutions of (1.19) are unique, there is no canonical inverse to the construction, and one cannot conclude uniqueness for the Yang-Mills flow. Kozono et. al. ([16], Theorem C) do prove that solutions in $H^{k}, k>>1$, are unique modulo gauge, as expected. Struwe, moreover, proves that as long as $D(t)$ is irreducible $\left(D(t): \Omega^{0}\left(\mathfrak{g}_{E}\right) \rightarrow \Omega^{1}\left(\mathfrak{g}_{E}\right)\right.$ has trivial kernel), then his weak solution is unique, including gauge.
} 
for all $x_{0} \in M$ and $0 \leq t<T$, then

$$
\|u(t)\|_{L^{2}} \leq e^{-\frac{t}{c R^{2}}}\|u(0)\|_{L^{2}}+C R^{-2}\left(1-e^{-\frac{2 t}{c R^{2}}}\right)^{1 / 2} \sup _{0 \leq s \leq t}\|u(s)\|_{L^{1}} .
$$

Proof. Let $\varphi \in C_{0}^{\infty}\left(B_{R}\left(x_{0}\right)\right)$. Multiplying (2.1) by $\varphi^{2} u$ and integrating by parts, we obtain

$$
\begin{aligned}
\frac{1}{2} \frac{d}{d t}\left(\int \varphi^{2} u^{2}\right)+\int \nabla\left(\varphi^{2} u\right) \cdot \nabla u & \leq A \int \varphi^{2} u^{5 / 2}+B \int \varphi^{2} u^{2} \\
\frac{1}{2} \frac{d}{d t}\left(\int \varphi^{2} u^{2}\right)+\int|\nabla(\varphi u)|^{2} & \leq \int|\nabla \varphi|^{2} u^{2}+A \int \varphi^{2} u^{5 / 2}+B \int \varphi^{2} u^{2} .
\end{aligned}
$$

Applying the Sobolev and Hölder inequalities on $B_{R}$ yields

$$
\frac{1}{2} \frac{d}{d t} \int \varphi^{2} u^{2}+\left(\frac{1}{C_{S}}-A \delta\right)\left(\int(\varphi u)^{4}\right)^{1 / 2} \leq\|\nabla \varphi\|_{L^{\infty}}^{2} \int_{B_{R}} u^{2}+B \int \varphi^{2} u^{2} .
$$

Provided that $R<R_{0}$, depending on the geometry of $M$, we may assume the following. First, that for any $x \in M$, the volume of any geodesic ball

$$
\operatorname{Vol}\left(B_{R}(x)\right) \leq c^{2} R^{4} .
$$

Second, that compactly supported functions on $B_{R}(x)$ obey a Sobolev inequality, with constant $C_{S}$ independent of $x$. Third, that it is possible to choose a cover of $M$ by geodesic balls $B_{R / 2}\left(x_{i}\right)$ in such a way that no more than $N$ of the balls $B_{i}=B_{R}\left(x_{i}\right)$ intersect a fixed ball, with $N$ universal in dimension four. For each $i$, let $\tilde{\varphi}_{i}$ be a cutoff for $B_{R / 2}\left(x_{i}\right) \subset B_{R}\left(x_{i}\right)$ with $\left\|\nabla \tilde{\varphi}_{i}\right\|_{L^{\infty}}<4 / R$, and define

$$
\varphi_{i}=\tilde{\varphi}_{i} / \sqrt{\sum \tilde{\varphi}_{j}^{2}}
$$

Then $\left\{\varphi_{i}^{2}\right\}$ is a partition of unity for $M$, with

$$
\left\|\nabla \varphi_{i}\right\|_{L^{\infty}}<C / R
$$

We now apply the above differential inequality with $\varphi=\varphi_{i}$, and sum on $i$, to obtain

$$
\begin{aligned}
\sum_{i}\left(\frac{1}{2} \frac{d}{d t} \int \varphi_{i}^{2} u^{2}+\left(C_{S}^{-1}-A \delta\right)\left(\int\left(\varphi_{i} u\right)^{4}\right)^{1 / 2}\right) & \leq \sum_{i}\left(C R^{-2} \int_{B_{i}}\left(\sum_{j} \varphi_{j}^{2}\right) u^{2}+B \int \varphi_{i}^{2} u^{2}\right) \\
& \leq\left(C N R^{-2}+B\right) \sum_{i} \int \varphi_{i}^{2} u^{2} .
\end{aligned}
$$

The interpolation inequality (7.10) of [12, with $p=1, q=2, r=4$, and $\mu=2$, yields

$$
\int\left(\varphi_{i} u\right)^{2} \leq 2 \theta\left(\int\left(\varphi_{i} u\right)^{4}\right)^{1 / 2}+2 \theta^{-2}\left(\int \varphi_{i} u\right)^{2} .
$$

Taking $\theta=R^{2} \delta$, we obtain

$$
\begin{aligned}
& \sum_{i}\left(\frac{d}{d t} \int \varphi_{i}^{2} u^{2}\right.\left.+2\left(C_{S}^{-1}-A \delta\right)\left(\int\left(\varphi_{i} u\right)^{4}\right)^{1 / 2}\right) \\
& \leq C\left(1+B R^{2}\right) \sum_{i}\left(\delta\left(\int\left(\varphi_{i} u\right)^{4}\right)^{1 / 2}+\delta^{-2} R^{-6}\left(\int \varphi_{i} u\right)^{2}\right) .
\end{aligned}
$$


Note that

$$
\sum_{i}\left(\int \varphi_{i} u\right)^{2} \leq\left(N \int u\right)^{2}
$$

Rearranging yields

$$
\sum_{i}\left(\frac{d}{d t} \int \varphi_{i}^{2} u^{2}+\epsilon\left(\int\left(\varphi_{i} u\right)^{4}\right)^{1 / 2}\right) \leq C \delta^{-2} R^{-6}\left(1+B R^{2}\right)\left(\int u\right)^{2}
$$

where

$$
\epsilon=2\left(C_{S}^{-1}-\delta\left(A+C\left(1+B R^{2}\right)\right)\right) .
$$

We now choose $R_{0}$ such that $B R_{0}^{2} \leq 1$, and let

$$
\delta=\left(2 C_{S}(A+2 C)\right)^{-1} .
$$

These choices ensure that $\epsilon \geq\left(C_{S}\right)^{-1}>0$. Hence we may apply Hölder's inequality to the left-hand side and absorb the partition of unity. This yields

$$
\frac{d}{d t} \int u^{2}+\frac{\epsilon}{c R^{2}} \int u^{2}=\sum_{i}\left(\frac{d}{d t} \int \varphi_{i}^{2} u^{2}+\frac{\epsilon}{c R^{2}} \int \varphi_{i}^{2} u^{2}\right) \leq C R^{-6}\left(\int u\right)^{2}
$$

and

$$
\frac{d}{d t}\left(e^{\frac{\epsilon}{c R^{2}} t} \int u(t)^{2}\right) \leq e^{\frac{\epsilon}{c R^{2}} t} C R^{-6}\left(\int u\right)^{2}
$$

Integrating in time, we obtain

$$
\int u(t)^{2} \leq e^{-\frac{\epsilon}{c R^{2}} t} \int u(0)^{2}+C \epsilon^{-1} R^{-4}\left(1-e^{-\frac{\epsilon}{c R^{2}} t}\right) \sup _{0 \leq s \leq t}\left(\int u(s)\right)^{2}
$$

which is equivalent to the desired bound.

Lemma 2.2. (Parabolic Moser iteration) Let $M$ be an $n$-dimensional Riemannian manifold and $B_{1} \subset M$ a unit geodesic ball. For $0<r \leq 1$, denote the parabolic cylinder

$$
P_{r}=B_{r} \times\left(-r^{2}, 0\right) .
$$

Fix

$$
0<p \leq \infty \quad \frac{n}{2}<q \leq \infty \quad K>0 \quad 0<\theta<1
$$

and assume that $u(x, t), f(x, t) \geq 0$ are functions on $P_{1}$ satisfying

$$
\left(\partial_{t}+\Delta\right) u \leq f \cdot u
$$

in the weak sense, with

$$
\sup _{-1<t<0}\|f(\cdot, t)\|_{L^{q}\left(B_{1}\right)} \leq K
$$

Then

$$
\|u\|_{L^{\infty}\left(P_{\theta}\right)} \leq C_{2.2}\|u\|_{L^{p}\left(P_{1}\right)} .
$$

The constant depends on $p, q, n, K, \theta, \operatorname{Vol}\left(B_{1}\right)$, and the Sobolev constant of $B_{1}$.

Proof. See Li [18], Lemma 19.1. 
Proposition 2.3. Let $R<\min \left(R_{0}, \sqrt{T}\right)$ and $u(t)$ be as in Proposition 2.1. Choose $\tau>0$ and let $\bar{\tau}=\tau / R^{2}$. For any $p \geq 1$ and $x_{0} \in M$, there holds

$$
\|u(t)\|_{L^{\infty}\left(B_{R / 2}\left(x_{0}\right)\right)} \leq C_{2.3} \sup _{t-\tau<s<t}\|u(s)\|_{L^{p}\left(B_{R}\left(x_{0}\right)\right)}
$$

for $0<\tau \leq t$, where $C 2.3=C\left(R^{-4}\left(1+\bar{\tau}^{-2}\right)\right)^{1 / p}$.

Proof. We use a well-known argument due to Schoen and Uhlenbeck [24]. After rescaling

$$
u(x, t) \rightarrow R^{4} u\left(R x, R^{2} t\right)
$$

in geodesic coordinates about $x_{0}$, we may assume that $u(x, t)$ is defined on $B_{1} \times[0,1]$. Then $u$ satisfies

$$
\begin{aligned}
\left(\partial_{t}+\Delta\right) u & \leq A u^{3 / 2}+R^{2} B u \\
& \leq\left(A u^{1 / 2}+1\right) \cdot u
\end{aligned}
$$

since we have chosen $R_{0}^{2} B \leq 1$, and

$$
\int_{B_{1}} u(t) \leq \delta^{2}
$$

As in the proof of Proposition 2.1, for $R<R_{0}$, the rescaled metric on $B_{1}$ (and any further rescaling) is close enough to Euclidean that we have uniform volume bounds and a uniform Sobolev constant $C_{S}$, hence may apply the previous Lemma.

Let $P_{r}(x, t)=B_{r}(x) \times\left[t-r^{2}, t\right]$, and abbreviate $P_{r}=P_{r}(0,1)$. Define

$$
e(r)=(1-r)^{4} \sup _{P_{r}} u
$$

and let $e_{0}, r_{0}$ be such that

$$
e_{0}=e\left(r_{0}\right)=\sup _{0 \leq r \leq 1} e(r) .
$$

Choose $\left(x_{1}, t_{1}\right) \in P_{r}$ such that $u\left(x_{1}, t_{1}\right)=\sup _{P_{r}} u$. Letting $\rho_{0}=\left(1-r_{0}\right) / 2$, we have

$$
\left(\rho_{0}\right)^{4} \sup _{P_{\rho_{0}}\left(x_{1}, t_{1}\right)} u \leq 16 e_{0} \text {. }
$$

Assume first that $e_{0}>1$. Letting

$$
\rho_{1}=\rho_{0}\left(e_{0}\right)^{-1 / 4}
$$

we may rescale

$$
u_{1}(x, t)=\left(\rho_{1}\right)^{4} u\left(\rho_{1} x+x_{1},\left(\rho_{1}\right)^{2}(t-1)+t_{1}\right)
$$

to obtain a function $u_{1}$ of $(x, t) \in P_{1}$. This again satisfies (2.3), and (2.6) implies

$$
\sup _{P_{1}} u_{1}(x, t) \leq 16
$$

But then Lemma 2.2, applied to (2.3) with $p=1$ and $q=\infty$, gives

$$
\begin{aligned}
1=u_{1}(0,1) & \leq C \int_{0}^{1} \int_{B_{1}} u_{1}(x, t) d V d t \\
& \leq C \delta^{2}
\end{aligned}
$$


for a constant depending only on $A$ (which is universal). For $\delta$ sufficiently small, this is a contradiction.

Therefore $e_{0} \leq 1$. Directly from the definition (2.4) and (2.5), we have for any $0<r<1$

$$
\sup _{P_{r}} u=(1-r)^{-4} e(r) \leq(1-r)^{-4} e_{0} \leq(1-r)^{-4} .
$$

We may therefore apply Lemma 2.2 to (2.3), to find

$$
\sup _{P_{1 / 2}} u \leq C\left(\int_{0}^{1} \int_{B_{3 / 4}} u^{p} d V d t\right)^{1 / p}
$$

If $\bar{\tau}<1$, we rescale by an additional factor $\sqrt{\bar{\tau}}$, and again obtain (2.8). Overall, undoing the rescaling, we have

$$
\|u(t)\|_{L^{\infty}\left(B_{R / 2}\left(x_{0}\right)\right)} \leq C\left(R^{-6}\left(1+\bar{\tau}^{-3}\right) \int_{t-\tau}^{t} \int_{B_{R}\left(x_{0}\right)} u(s)^{p} d V d s\right)^{1 / p}
$$

for $t \geq \tau$. The desired estimate follows directly from (2.9) with $\bar{\tau} \leq 1$.

\subsection{Criterion for long-time existence.}

Lemma 2.4. (C.f. [10], 7.2.10). There is a universal constant $C$ and for any $N \geq 2, R>0$, a smooth radial function $\beta=\beta_{N, R}$ on $\mathbb{R}^{4}$, with

$$
\begin{gathered}
0 \leq \beta(x) \leq 1 \\
\beta(x)= \begin{cases}1 & |x| \leq R / N \\
0 & |x| \geq R\end{cases}
\end{gathered}
$$

and

$$
\|\nabla \beta\|_{L^{4}}+\left\|\nabla^{(2)} \beta\right\|_{L^{2}}<\frac{C}{\sqrt{\log N}} .
$$

Assuming $R<R_{0}$, the same holds for $\beta\left(x-x_{0}\right)$ on any geodesic ball $B_{R}\left(x_{0}\right) \subset M$.

Proof. We take

$$
\beta(x)=\tilde{\varphi}\left(\frac{\log \frac{N}{R}|x|}{\log N}\right)
$$

where

$$
\tilde{\varphi}(s)= \begin{cases}1 & s \leq 0 \\ 0 & s \geq 1\end{cases}
$$

is a standard cutoff function, with respect to the cylindrical coordinate $s$.

Theorem 2.5. Assume that $A(t)$ is a smooth solution of the Yang-Mills flow on $M$ for $0 \leq t<T$, and write $F(t)=F_{A(t)}$. Let $B_{R / N} \subset B_{R}$ be concentric geodesic balls in $M$, with $R<R_{0}$ and $N \geq 2$. There holds

$$
\|F(t)\|_{L^{2}\left(B_{R / N}\right)}^{2} \leq\|F(0)\|_{L^{2}\left(B_{R}\right)}^{2}+\int_{0}^{t} \frac{\left\|F^{+}(s)\right\|_{L^{\infty}\left(B_{R}\right)}}{\sqrt{\log (N)}}\left(C+\left\|F^{-}(s)\right\|_{L^{2}\left(B_{R}\right)}^{2}\right) d s .
$$


Therefore, if

$$
\left\|F^{+}(t)\right\|_{L^{\infty}(M)} \in L^{1}([0, T))
$$

or, in particular, if $F^{+}$does not concentrate in $L^{2}$ as $t \rightarrow T$, then $\lim _{t \rightarrow T} A(t)$ exists in $C^{\infty}(M)$ and the flow extends smoothly.

Proof. Recall the evolution of the curvature tensor

$$
\frac{\partial}{\partial t} F+D D^{*} F=0
$$

Taking an inner-product with $\varphi^{2} F$ and integrating by parts, we obtain

$$
\frac{1}{2} \frac{d}{d t} \int \varphi^{2}|F|^{2} d V+\int\left\langle D^{*}\left(\varphi^{2} F\right), D^{*} F\right\rangle d V=0 .
$$

Note from (1.7) that

$$
\begin{aligned}
D^{*}\left(\varphi^{2} F\right)_{j} & =-\nabla^{k}\left(\varphi^{2} F_{k j}\right) \\
& =-2 \varphi \nabla^{k} \varphi F_{k j}+\varphi^{2} D^{*} F_{j} .
\end{aligned}
$$

Abbreviating $\|\cdot\|=\|\cdot\|_{L^{2}(M)}$ as before, we therefore have

$$
\frac{1}{2} \frac{d}{d t}\|\varphi F\|^{2}+\left\|\varphi D^{*} F\right\|^{2}=2 \int\left\langle\varphi \nabla^{k} \varphi F_{k j}, D^{*} F^{j}\right\rangle d V .
$$

On the right-hand side we substitute $D^{*} F=2 D^{*} F^{+}$, and integrate by parts again, to obtain

$$
\frac{1}{2} \frac{d}{d t}\|\varphi F\|^{2}+\left\|\varphi D^{*} F\right\|^{2}=4 \int\left\langle\left(\nabla_{i} \varphi \nabla^{k} \varphi+\varphi \nabla_{i} \nabla^{k} \varphi\right) F_{k j}+\varphi \nabla^{k} \varphi \nabla_{i} F_{k j},\left(F^{+}\right)^{i j}\right\rangle d V .
$$

In the inner product with the self-dual two-form $F^{+}$, we may replace the term $\varphi \nabla^{k} \varphi \nabla_{i} F_{k j}$ via the identity

$$
\begin{aligned}
\left(\nabla^{k} \varphi\left(\nabla_{i} F_{k j}-\nabla_{j} F_{k i}\right)\right)^{+} & =\left(\nabla^{k} \varphi\left(\left(-\nabla_{j} F_{i k}-\nabla_{k} F_{j i}\right)-\nabla_{j} F_{k i}\right)\right)^{+} \\
& =\left(\nabla^{k} \varphi \nabla_{k} F_{i j}\right)^{+} \\
& =\nabla^{k} \varphi \nabla_{k} F_{i j}^{+}
\end{aligned}
$$

We then write

$$
\left\langle\nabla_{k} F_{i j}^{+},\left(F^{+}\right)^{i j}\right\rangle=\frac{1}{2} \nabla_{k}\left|F^{+}\right|^{2}
$$

and integrate by parts once more, to obtain

$$
\begin{aligned}
\frac{1}{2} \frac{d}{d t}\|\varphi F\|^{2}+\left\|\varphi D^{*} F\right\|^{2} & =4 \int\left(\nabla_{i} \varphi \nabla_{k} \varphi+\varphi \nabla_{i} \nabla_{k} \varphi\right)\left(\left\langle F^{k}{ }_{j},\left(F^{+}\right)^{i j}\right\rangle-g^{i k} \frac{\left|F^{+}\right|^{2}}{4}\right) d V \\
& =4 \int\left(\nabla_{i} \varphi \nabla_{k} \varphi+\varphi \nabla_{i} \nabla_{k} \varphi\right)\left\langle\left(F^{-}\right)^{k}{ }_{j},\left(F^{+}\right)^{i j}\right\rangle d V
\end{aligned}
$$

where the second identity follows from a calculation as in Section 1.5. Removing an $L^{\infty}$ norm, and applying Young's inequality, yields

$$
\frac{d}{d t}\|\varphi F\|^{2} \leq 8\left\|F^{+}\right\|_{L^{\infty}\left(B_{r}\right)}\left(\epsilon^{-1}\left\|F^{-}\right\|_{L^{2}}^{2}+\epsilon\left(\|\nabla \varphi\|_{L^{4}}^{4}+\left\|\varphi \nabla^{2} \varphi\right\|_{L^{2}}^{2}\right)\right) .
$$


Choose $\epsilon=8 \sqrt{\log (N)}$ and $\varphi=\beta_{N, r}$, from Lemma 2.4 to obtain the desired estimate (2.10).

By Theorem 1.2, to prove the second claim, it suffices to show that the full curvature does not concentrate in $L^{2}$ at time $T$. Note that $\left\|F^{-}(t)\right\|_{L^{2}\left(B_{R}\right)}^{2}$ is bounded above by the total anti-self-dual energy, which by (1.12) is decreasing. Therefore if the full curvature on $B_{R}$ is initially less than $\epsilon_{0} / 2$, then for $N$ sufficiently large, (2.10) implies that the full curvature on $B_{R / N}$ will remain less than $\epsilon_{0}$ at time $T$.

Lastly, by Proposition 2.3 , non-concentration of $F^{+}$implies a uniform $L^{\infty}$ bound, and hence the required $L^{1}\left(L^{\infty}\right)$ bound on the self-dual energy at finite time.

Corollary 2.6. Let $\delta$ be the universal constant of Proposition [2.3. If an initial connection has self-dual curvature $\left\|F^{+}\right\|_{L^{2}(M)}<\delta$, then the Yang-Mills flow exists for all time, with curvature blowing up at most exponentially as $t \rightarrow \infty$.

Corollary 2.7. If the maximal existence time, $T$, is finite, then both $F^{+}$and $F^{-}$must concentrate at some point $x_{0} \in M$ as $t \rightarrow T$.

Remark 2.8. The proof of Theorem 2.5 gives a more refined long-time existence criterion, which will be the basis for a forthcoming paper. Let

$$
S_{i j}=\left\langle F_{i}^{k}, F_{j k}\right\rangle-\frac{1}{4}|F|^{2} g_{i j}=2\left\langle F_{i}^{+k}, F_{j k}^{-}\right\rangle
$$

be the stress-energy tensor for Yang-Mills, and define

$$
N(x, t)=x^{i} x^{j} S_{i j}
$$

Here $x^{i}$ are geodesic coordinates centered at $x_{0} \in M$.

Theorem 2.9. If, for some $r_{0}>0$, there holds

$$
\lim _{t \rightarrow T} \sup _{0<r<r_{0}}\left|f_{S_{r}^{3}\left(x_{0}\right)} N(x, t) d S_{x}\right|<\infty
$$

then no singularity occurs at $\left(x_{0}, T\right)$.

\section{Convergence at infinite time}

We now turn to the question of convergence of the Yang-Mills flow as $t \rightarrow \infty$, making the assumption

$$
\left\|F_{A}^{+}\right\|_{L^{2}(M)}<\delta .
$$

By (1.12), this condition is preserved by the flow, which, according to Corollary 2.6, exists for all time. Moreover, if $E$ has structure group $S U(2)$, then (3.1) should represent the generic end-behavior [2].

We are particularly concerned with the interplay between weak Uhlenbeck limits, which are taken modulo gauge away from bubble points, and smooth convergence of the flow. Lemma 3.4 provides a rapid proof in the present context, as in that of Kahler surfaces, that the former always exist and are Yang-Mills. Because of the $(T-\tau)$ factor in the estimates of Proposition 3.3, however, fast decay of the energy is needed in order to conclude that the 
flow converges smoothly A cohomological assumption on the Uhlenbeck limit will supply the required estimate (3.12) along the flow.

For the remainder of the paper, $\Omega \subset M$ will denote an open set, and we let

$$
\Omega_{r}=\left\{x \in \Omega \mid d\left(x, \Omega^{c}\right)>r\right\} \subset \subset \Omega .
$$

For points $x_{1}, \ldots, x_{m} \in M$, write

$$
M_{0}=M \backslash\left\{x_{1}, \ldots, x_{m}\right\}
$$

and

$$
M_{r}=\left(M_{0}\right)_{r}=M \backslash \bar{B}_{r}\left(x_{1}\right) \cup \cdots \cup \bar{B}_{r}\left(x_{m}\right) .
$$

As before, we will abbreviate $\|\cdot\|=\|\cdot\|_{L^{2}(M)}$.

\subsection{Basic estimates.}

Lemma 3.1. ( $\epsilon$-regularity) There exists $\epsilon_{0}>0$ as follows. For $R<R_{0}$, if

$$
\|F(t)\|_{L^{2}\left(B_{R}\right)}^{2}<\epsilon_{0}
$$

for all times $t$ with $-R^{2} \leq t<0$, then there holds

$$
\left\|\nabla_{A}^{(k)} F(t)\right\|_{L^{\infty}\left(B_{R_{k}}\right)}<\frac{93.1}{R^{2+k}}
$$

for $k \geq 0$ and $-R_{k}^{2} \leq t<0$, where $R_{k}=R / 2^{k+1}$. The constant depends only on $k$.

Proof. The $k=0$ bound follows from Proposition 2.3, with $p=1$ and $\bar{\tau}=1.8$ For $k \geq 1$, this is the result of the Bernstein-Hamilton-type derivative estimates of [35], Theorem 2.2.

Proposition 3.2. Assume $\|F(t)\|_{L^{\infty}\left(B_{R}\left(x_{0}\right)\right)}<K$ for $0 \leq t<T$. Then for $\tau>0, R<R_{0}$, we have

$$
\begin{aligned}
\left\|\nabla^{(k)} D^{*} F(t)\right\|_{L^{\infty}\left(B_{R_{k}}\right)}^{2} & \leq C 3.2\left\|D^{*} F\right\|_{L^{2}\left(B_{R} \times[t-\tau, t]\right)}^{2} \\
\left\|\nabla^{(k)} F(t)\right\|_{L^{\infty}\left(B_{R_{k}}\right)}^{2} & \leq C 3.2\left(\left\|D^{*} F\right\|_{L^{2}\left(B_{R} \times[t-\tau, t]\right)}^{2}+\|F\|_{L^{2}\left(B_{R} \times[t-\tau, t]\right)}^{2}\right)
\end{aligned}
$$

for $k \geq 0$ and $k \tau \leq t<T$. The constants depend on $K, k, R$, and $\tau$.

Proof. One computes the evolution

$$
\begin{aligned}
\frac{\partial}{\partial t}\left(D^{*} F\right)_{i} & =-\frac{\partial}{\partial t} \nabla^{k} F_{k i} \\
& =-\left[\left(-D^{*} F\right)^{k}, F_{k i}\right]-\left(D^{*} D D^{*} F\right)_{i} \\
& =\left[F_{i}^{k},\left(D^{*} F\right)_{k}\right]-\left(\Delta_{A} D^{*} F\right)_{i} \\
& =\nabla^{k} \nabla_{k} D^{*} F_{i}+2\left[F_{i}^{k}, D^{*} F_{k}\right]+R m \# D^{*} F .
\end{aligned}
$$

In the third line, we used the identity (1.9) to obtain the Hodge Laplacian. Multiplying (3.3) by $D^{*} F$ gives

$$
\left(\partial_{t}+\Delta\right)\left|D^{*} F\right|^{2} \leq C(1+K)\left|D^{*} F\right|^{2} .
$$

\footnotetext{
${ }^{7}$ This subtlety also obstructs an obvious proof of long-time existence using only Uhlenbeck compactness and $\epsilon$-regularity to produce a bubble tree and control it.

${ }^{8}$ Via the monotonicity formula [14, it suffices to assume (3.2) only at $t=-R^{2}$ (see [4], 15]).
} 
The first estimate, with $k=0$, follows from Lemma 2.2 applied to (3.3). Applying a cutoff for $B_{3 R_{1} / 2} \subset B_{R_{0}}$ and using Young's inequality, as well as the $k=0$ estimate, one also obtains

$$
\int_{t-\tau / 2}^{t}\left\|\nabla D^{*} F(s)\right\|_{L^{2}\left(B_{3 R_{1} / 2}\right)}^{2} d s \leq C\left\|D^{*} F\right\|_{L^{2}\left(B_{R} \times[t-\tau, t]\right)}^{2} .
$$

Next, applying $\nabla$ to (3.3), one obtains an evolution equation

$$
\left(\partial_{t}+\nabla^{*} \nabla\right) \nabla D^{*} F=F \# \nabla D^{*} F+R m \# \nabla D^{*} F+\nabla F \# D^{*} F+\nabla R m \# D^{*} F .
$$

Note from Lemma 3.1 that all derivatives of $F$ are bounded in terms of $K$. Multiplying (3.5) by $\nabla D^{*} F$ and again applying Lemma 2.2 , we bound $\left\|\nabla D^{*} F(t)\right\|_{L^{\infty}\left(B_{R_{1}}\right)}$, for $t \geq \tau$, by the LHS of (3.4), which concludes the $k=1$ case. The higher derivative estimates proceed by induction, using formulae similar to (3.5).

The argument for the second estimate is identical, beginning with the inequality

$$
\|\nabla F\|_{L^{2}\left(B_{R_{0}}\right)}^{2} \leq C\left(\left\|D^{*} F\right\|_{L^{2}\left(B_{R}\right)}^{2}+\|F\|_{L^{2}\left(B_{R}\right)}^{2}\right)
$$

which follows from the Weitzenbock formula (1.13).

Proposition 3.3. Let $0<\tau_{0} \leq \tau<T$, and assume $\|F(t)\|_{L^{\infty}(\Omega)}<K$ for $\tau-\tau_{0} \leq t<T$. Then we have the $L^{\infty}$ bound

$$
\|A(T)-A(\tau)\|_{L^{\infty}\left(\Omega_{r}\right)}^{2} \leq C_{3.3}\left(\left\|F\left(\tau-\tau_{0}\right)\right\|^{2}-\|F(T)\|^{2}\right)(T-\tau)
$$

as well as the Sobolev bounds

$$
\begin{aligned}
\|A(T)-A(\tau)\|_{H^{k}\left(\Omega_{r}\right)}^{2} \leq C \overline{3.3}\left(\left\|F\left(\tau-\tau_{0}\right)\right\|^{2}-\|F(T)\|^{2}\right)(T-\tau) \\
\cdot\left(1+\sup _{\tau \leq t<T}\|A(t)\|_{H^{k-1}(\Omega)}^{2 k}\right)
\end{aligned}
$$

for $k \geq 1$. The constants depend on $K, k, r, \tau_{0}, \Omega \subset M$, and $\nabla_{\text {ref }}($ for $k \geq 1$ ).

Proof. For the first bound, we calculate

$$
\begin{aligned}
\|A(T)-A(\tau)\|_{L^{\infty}\left(\Omega_{r}\right)} & \leq \int_{\tau}^{T}\left\|D^{*} F(t)\right\|_{L^{\infty}\left(\Omega_{r}\right)} d t \\
& \leq C \overline{3.2} \int_{\tau}^{T}\left\|D^{*} F\right\|_{L^{2}\left(\Omega \times\left[t-\tau_{0}, t\right]\right)} d t \\
& \leq C(T-\tau)^{1 / 2}\left(\int_{\tau}^{T}\left\|D^{*} F\right\|_{L^{2}\left(\Omega \times\left[t-\tau_{0}, t\right]\right)}^{2} d t\right)^{1 / 2} \\
& \leq C(T-\tau)^{1 / 2}\left(\int_{\tau}^{T} \int_{t-\tau_{0}}^{t}\left\|D^{*} F(s)\right\|^{2} d s d t\right)^{1 / 2} .
\end{aligned}
$$

The domain of integration

$$
t-\tau_{0} \leq s \leq t \quad \tau \leq t \leq T
$$

may be relaxed to

$$
\tau-\tau_{0} \leq s \leq T \quad s \leq t \leq s+\tau_{0}
$$


Then (3.7) becomes

$$
\begin{aligned}
\|A(T)-A(\tau)\|_{L^{\infty}\left(\Omega_{r}\right)} & \leq C(T-\tau)^{1 / 2} \tau_{0}^{1 / 2}\left(\int_{\tau-\tau_{0}}^{T}\left\|D^{*} F(s)\right\|^{2} d s\right)^{1 / 2} \\
& \leq C(T-\tau)^{1 / 2}\left(\left\|F\left(\tau-\tau_{0}\right)\right\|^{2}-\|F(T)\|^{2}\right)^{1 / 2}
\end{aligned}
$$

as desired. For $k=1$, write

$$
\begin{aligned}
\partial_{t} \nabla_{r e f} A & =-\nabla_{r e f} D^{*} F \\
& =-\nabla_{A} D^{*} F+A \# D^{*} F .
\end{aligned}
$$

We then have

$$
\left\|\nabla_{\text {ref }}(A(T)-A(\tau))\right\|_{L^{2}} \leq C\left(1+\sup \|A\|_{L^{2}}\right) \int_{\tau}^{T}\left(\left\|D^{*} F(t)\right\|_{L^{\infty}}+\left\|\nabla_{A} D^{*} F(t)\right\|_{L^{\infty}}\right) d t
$$

and may apply Proposition 3.2 as above. For $k=2$, write

$$
\begin{aligned}
\partial_{t} \nabla_{r e f}^{(2)} A & =-\nabla_{r e f}^{(2)} D^{*} F \\
& =\nabla_{A}^{(2)} D^{*} F+A \# \nabla_{A} D^{*} F+\nabla_{r e f} A \# D^{*} F+A \# A \# D^{*} F
\end{aligned}
$$

and note that $\|A\|_{L^{2}}+\left\|\nabla_{\text {ref }} A\right\|_{L^{2}}+\|A \# A\|_{L^{2}} \leq C\left(1+\|A\|_{H^{1}}^{2}\right)$. The higher derivative bounds proceed similarly.

3.2. Uhlenbeck limits. For a sequence $t_{j} \rightarrow \infty$, we say that $\left(E_{\infty}, A_{\infty}\right)$ is an Uhlenbeck limit along the flow if there exists a subsequence of times $t_{j_{k}}$ and smooth bundle isometries $u_{k}: E \rightarrow E_{\infty}$, defined on an exhaustion of open sets

$$
U_{1} \subset \cdots \subset U_{k} \subset \cdots \subset M_{0}=M \backslash\left\{x_{1}, \ldots, x_{m}\right\}
$$

such that

$$
u_{k}\left(A\left(t_{j_{k}}\right)\right) \rightarrow A_{\infty}
$$

smoothly on any $\Omega \subset \subset M_{0}$.

Lemma 3.4. Assume $\left\|F^{+}(t)\right\|_{L^{\infty}(\Omega)}<K^{+}$for $0 \leq t \leq \tau$. Let $\epsilon_{0}$ be as in Lemma 3.1, and assume that for some $0<r_{0}<R_{0}$ there holds

$$
\|F(\tau)\|_{L^{2}\left(B_{r_{0}}\left(x_{0}\right)\right)}^{2}<\epsilon_{0} / 3
$$

for all $x_{0} \in \Omega_{r_{0}}$, with $0<r_{0}^{2}<\tau$. If

$$
\|F(0)\|_{L^{2}(M)}^{2}-\|F(\tau)\|_{L^{2}(M)}^{2} \leq \epsilon_{0} / 3
$$

then we have

$$
\left\|\nabla_{A}^{(k)} F(\tau)\right\|_{L^{\infty}\left(\Omega_{r_{0}}\right)}<\frac{93.4}{r_{0}^{2+k}}
$$

for $k \geq 0$. The constant depends on $K^{+},\|F(0)\|$, and $k$. 
Proof. Let $x_{0} \in \Omega_{r_{0}}$, and $\varphi$ be the cutoff of Lemma 2.4 for $B_{r_{0} / N}\left(x_{0}\right) \subset B_{r_{0}}\left(x_{0}\right)$. We apply the proof of Theorem 2.5 using $\bar{\varphi}=1-\varphi$. This gives

$$
\|F(\tau)\|_{L^{2}\left(M \backslash B_{r_{0}}\right)}^{2}-\|F(t)\|_{L^{2}\left(M \backslash B_{r_{0} / N}\right)}^{2}<\epsilon_{0} / 3
$$

for $N$ large enough based on $\|F\|^{2}$ and $K^{+}$, but independent of $x_{0}$ and $r_{0}$. Adding (3.9), with $t$ in place of zero, and (3.10), we obtain

$$
\|F(t)\|_{L^{2}\left(B_{r_{0} / N}\right)}^{2}-\|F(\tau)\|_{L^{2}\left(B_{r_{0}}\right)}^{2}<2 \epsilon_{0} / 3 .
$$

By (3.8), we have

$$
\|F(t)\|_{L^{2}\left(B_{r_{0} / N}\right)}^{2}<\epsilon_{0}
$$

for $0 \leq t \leq \tau$. The desired bounds follow from Lemma 3.1 .

Theorem 3.5. Assume $\left\|F^{+}(t)\right\|_{L^{\infty}(M)}<K^{+}$for $t$ sufficiently large. For any sequence $t_{j} \rightarrow \infty$, there exists an Uhlenbeck limit, and any such limit is Yang-Mills.

Proof. This is a direct adaptation of the arguments contained in Donaldson and Kronheimer [10], $\S 4$ and $\S 6.2 .4$, as follows. The existence of weak $H^{1}$ limits on a countable family of balls in $M_{0}$ is the result of compactness theory for connections with bounded $L^{2}$ curvature in Coulomb gauge ([26], 32]). By Lemma 3.4, we in fact have $L^{\infty}$ bounds on the curvature of $A\left(t_{j_{k}}\right)$ and all its derivatives on each ball, for $k$ large enough. By [10], Lemma 2.3.11, the weak limits can be taken to be smooth limits over each ball; and by [10, Corollary 4.4.8, the gauge transformations can be patched together over the open sets $U_{i} \cdot 9$

The fact that the limiting connection is Yang-Mills away from the bubbling points, and therefore extends to a smooth Yang-Mills connection on $E_{\infty}$, follows directly from the energy inequality, Proposition [3.2, and [33].

3.3. Sobolev and Poincaré inequality for self-dual forms. The following brief discussion amounts to the key analytic observation of Taubes [30] (see also [10], §7).

Assuming $\left\|F_{A}^{+}\right\|<\delta$, Hölder's inequality applied to the Weitzenbock formula (1.14) implies, for $\omega \in \Omega^{2+}\left(\mathfrak{g}_{E}\right)$, the Sobolev inequality

$$
\begin{aligned}
\|\omega\|_{L^{4}(M)}^{2}+\left\|\nabla_{A} \omega\right\|^{2} & \leq C_{M}\left(\left\|D_{A} \omega\right\|^{2}+\left\|D_{A}^{*} \omega\right\|^{2}+\|\omega\|^{2}\right) \\
& \leq C_{M}\left(\left\|D_{A}^{*} \omega\right\|^{2}+\|\omega\|^{2}\right) .
\end{aligned}
$$

In the second line, we used the pointwise identity

$$
\left|D_{A} \omega\right|=\left|-* D_{A} * \omega\right|=\left|D_{A}^{*} \omega\right| .
$$

In case $A$ is an instanton, recall the basic complex ([10], §4.2.5)

$$
0 \longrightarrow \mathfrak{g}_{E} \stackrel{D_{A}}{\longrightarrow} \Omega^{1}\left(\mathfrak{g}_{E}\right) \stackrel{D_{A}^{+}}{\longrightarrow} \Omega^{2+}\left(\mathfrak{g}_{E}\right) \longrightarrow 0 .
$$

\footnotetext{
${ }^{9}$ Note that Theorem 1.3(ii) of Schlatter 22, which finds a Yang-Mills connection as a weak limit along a specially chosen sequence of times, does not include any patching, as this may not be possible with $H^{2}$ gauge transformations.
} 
The assumption of vanishing second cohomology group, which we will write $H_{A}^{2+}=0$, is equivalent to the statement

$$
D_{A}^{*} \omega=0 \Longrightarrow \omega=0 \text {. }
$$

The usual compactness argument then gives an inequality

$$
\|\omega\|^{2} \leq C_{A}\left\|D_{A}^{*} \omega\right\|^{2}
$$

for $\omega \in \Omega^{2+}\left(\mathfrak{g}_{E}\right)$. Hence this term can be dropped from the RHS of (3.11), yielding

$$
\|\omega\|_{L^{4}}^{2}+\|\omega\|^{2}+\left\|\nabla_{A} \omega\right\|^{2} \leq C_{A}\left\|D_{A}^{*} \omega\right\|^{2} .
$$

We require only the Poincaré inequality

$$
\|\omega\|_{L^{4}}^{2}+\|\omega\|^{2} \leq C_{A}\left\|D_{A}^{*} \omega\right\|^{2}
$$

for $\omega \in \Omega^{2+}\left(\mathfrak{g}_{E}\right)$, where we always take $C_{A} \geq C_{M}$. Here $A$ need not be an instanton. This inequality has the following basic stability property in dimension four.

Lemma 3.6. Fix $x_{1}, \ldots, x_{m} \in M$, and let $A_{0}$ be a connection on a bundle $E_{0} \rightarrow M$ which satisfies (3.12) with constant $C_{0}=C_{A_{0}}$. Assume that $A$ is a connection on $E$ with $\left\|F_{A}^{+}\right\|<\delta$, for which there exists a smooth bundle isometry $u: E_{0} \rightarrow E$ defined over $M_{r}=M \backslash \bar{B}_{r}\left(x_{1}\right) \cup$ $\cdots \cup \bar{B}_{r}\left(x_{m}\right)$, such that

$$
\left\|u(A)-A_{0}\right\|_{L^{4}\left(M_{r}\right)} \leq \epsilon .
$$

If $\epsilon$ and $r$ are sufficiently small, depending only on $C_{0}, R_{0}$, and $m$, then $A$ satisfies (3.12) with constant $8 C_{0}$.

Proof. Assume first that $\operatorname{Supp}(\omega) \subset M_{r}$. Write $\tilde{A}=u(A), \tilde{\omega}=u(\omega), a=A_{0}-\tilde{A}$. We then have

$$
\left\|D_{A}^{*} \omega\right\|^{2}=\left\|D_{\tilde{A}}^{*} \tilde{\omega}\right\|^{2}=\left\|D_{A_{0}}^{*} \tilde{\omega}+a \# \tilde{\omega}\right\|^{2}
$$

and

$$
\left\|D_{A_{0}}^{*} \tilde{\omega}\right\|^{2} \leq 2\left(\left\|D_{A}^{*} \omega\right\|^{2}+\|a\|_{L^{4}}^{2}\|\omega\|_{L^{4}}^{2}\right) .
$$

On the other hand, if $\operatorname{Supp}(\omega) \subset B_{r}\left(x_{1}\right) \cup \cdots \cup B_{r}\left(x_{m}\right)$, then

$$
\|\omega\|^{2} \leq c m r^{2}\|\omega\|_{L^{4}}^{2}
$$

Choose $\epsilon, r, N$ such that

$$
4 \epsilon^{2}+c m r^{2}+C / \log (N)<\left(8 C_{0}\right)^{-1} .
$$

Let $\varphi=\sum \beta_{N, r}\left(x-x_{i}\right)$ be a sum of the logarithmic cutoffs of Lemma 2.4, and $\bar{\varphi}=1-\varphi$. Combining the above observations, we have

$$
\begin{aligned}
\|\omega\|_{L^{4}}^{2}+\|\omega\|^{2} & \leq 2\left(\|\varphi \omega\|_{L^{4}}^{2}+\|\varphi \omega\|^{2}+\|\bar{\varphi} \omega\|_{L^{4}}^{2}+\|\bar{\varphi} \omega\|^{2}\right) \\
& \leq 2 C_{M}\left(\left\|D_{A}^{*}(\varphi \omega)\right\|^{2}+\|\varphi \omega\|^{2}\right)+2 C_{0}\left\|D_{A_{0}}^{*}(\bar{\varphi} \tilde{\omega})\right\|^{2} \\
& \leq 4 C_{0}\left(\left\|\varphi D_{A}^{*} \omega\right\|^{2}+\left\|\bar{\varphi} D_{A}^{*} \omega\right\|^{2}+2\|D \varphi \# \omega\|^{2}+\left(4\|a\|_{L^{4}}^{2}+c m r^{2}\right)\|\omega\|_{L^{4}}^{2}\right) \\
& \leq 4 C_{0}\left(\left\|D_{A}^{*} \omega\right\|^{2}+\left(2\|D \varphi\|_{L^{4}}^{2}+4 \epsilon^{2}+c m r^{2}\right)\|\omega\|_{L^{4}}^{2}\right) .
\end{aligned}
$$

Rearranging yields the desired estimate, where we replace $r / N$ by $r$. 


\subsection{Convergence.}

Theorem 3.7. Fix $x_{1}, \ldots, x_{m} \in M$, and let $A_{0}, E_{0}, \epsilon$, and $r$ be as in Lemma 3.6. Choose $\tau_{0}>0$ and $0<r_{0}<\min \left(r / 3, R_{0}, \sqrt{\tau_{0}}\right)$. There exists $\bar{\delta}_{1}>0$ as follows.

Assume that $A(t)$ solves the Yang-Mills flow with $\left\|F_{A(0)}^{+}\right\|=\left\|F^{+}(0)\right\|<\delta$, and for some $\tau \geq \tau_{0}$, there hold

$$
\begin{gathered}
\left\|F^{+}\left(\tau-\tau_{0}\right)\right\| \leq \delta_{1}<\bar{\delta}_{1} \\
\|F(\tau)\|_{L^{2}\left(B_{r_{0}}(x)\right)}<\epsilon_{0} / 3 \quad \forall x \in M_{2 r / 3}
\end{gathered}
$$

and, for a bundle isometry $u: E \rightarrow E_{0}$ over $M_{r / 3}$

$$
\left\|u(A(\tau))-A_{0}\right\|_{L^{4}\left(M_{r / 3}\right)} \leq \epsilon_{1}<\epsilon / 2 .
$$

Then the flow converges smoothly on $M$ as $t \rightarrow \infty$, with limit an instanton $A_{\infty}$ on $E$. We have also the bounds

$$
\begin{gathered}
\left\|u\left(A_{\infty}\right)-A_{0}\right\|_{L^{4}\left(M_{r}\right)} \leq C 3.7 \delta_{1}+\epsilon_{1} \\
\left\|A(t)-A_{\infty}\right\|_{L^{\infty}\left(M_{r}\right)} \leq C 3.7 \delta_{1} e^{-(t-\tau) / C_{1}}
\end{gathered}
$$

for $t \geq \tau$, and

$$
\left\|A(t)-A_{\infty}\right\|_{H^{k}\left(M_{r}\right)} \leq C 3.7 \delta_{1} e^{-(t-\tau) / C_{1}}\left(1+\|A(\tau)\|_{H^{k-1}\left(M_{r / 2}\right)}^{k !}\right)
$$

for $k \geq 1$.

The constants 3.7 and $\bar{\delta}_{1}$ depend on $C_{A_{0}}, r_{0}, \tau_{0}, m, k, \kappa(E), \nabla_{\text {ref }}$ (for $k \geq 1$ ), and the geometry of $M$. The constant $C_{1}$ is a universal multiple of $C_{A_{0}}$.

Proof. Recall, from Corollary 2.6, that the solution $A(t)$ exists for all time and is smooth. By Lemma 3.6, the inequality

$$
\left\|F^{+}(t)\right\|^{2} \leq 8 C_{A_{0}}\left\|D^{*} F^{+}(t)\right\|^{2}
$$

holds for $A(t)$ on some maximal time interval $\tau \leq t<T$. We will argue that if $\delta_{1}$ is sufficiently small, then $T=\infty$ and the flow converges. Assume, for contradiction, that $T<\infty$.

Applied to the global energy inequality, (3.16) yields

$$
\frac{d}{d t}\left\|F^{+}\right\|^{2}+C_{1}^{-1}\left\|F^{+}\right\|^{2} \leq \frac{d}{d t}\left\|F^{+}\right\|^{2}+4\left\|D^{*} F^{+}\right\|^{2}=0 .
$$

In view of (3.13), this implies the exponential bound

$$
\frac{1}{2}\left(\|F(t)\|^{2}-\|F(T)\|^{2}\right) \leq\left\|F^{+}(t)\right\|^{2} \leq \delta_{1}^{2} e^{-(t-\tau) / C_{1}}
$$

for $\tau-\tau_{0} \leq t \leq T$. Proposition 2.3, with $p=1$, then implies the global $L^{\infty}$ bound

$$
\left\|F^{+}(t)\right\|_{L^{\infty}(M)}^{2} \leq C_{2.3} \delta_{1}^{2} e^{-(t-\tau) / C_{1}}=: K^{+}(t)^{2} .
$$

Therefore, if $\delta_{1}$ is sufficiently small, we have

$$
\left(C+\|F(0)\|^{2}\right) \int_{\tau}^{T} K^{+}(t) d t<\epsilon_{0} / 3
$$


By Theorem 2.5 and (3.14), the full curvature cannot concentrate on $M_{2 r / 3}$ before time $T$, and we have a uniform bound

$$
\|F(t)\|_{L^{\infty}\left(M_{2 r / 3}\right)}<K
$$

for $\tau+r_{0}^{2}<t<T$.

In order to apply Proposition 3.3 , we need this curvature bound on $M_{2 r / 3}$ also from time $\tau-r_{0}^{2} / 2$. Note that

$$
\delta_{1}^{2} \geq\left\|F^{+}\left(\tau-r_{0}^{2}\right)\right\|^{2} \geq \frac{1}{2}\left(\left\|F\left(\tau-r_{0}^{2}\right)\right\|^{2}-\|F(T)\|^{2}\right) .
$$

Hence, provided that we choose $\delta_{1}^{2}<\epsilon_{0} / 6$ and enlarge $K$ appropriately, Lemma 3.4 gives a uniform bound of the form (3.20) for $\tau-r_{0}^{2} / 2<t \leq \tau+r_{0}^{2}$.

We may now apply Proposition 3.3 and (3.17) at each time $\tau+i$, to conclude

$$
\|A(\tau+i+1)-A(\tau+i)\|_{L^{\infty}\left(M_{r}\right)}^{2} \leq C 3.3\left(K^{+}(\tau+i)\right)^{2} .
$$

By the triangle inequality and geometric series, we have

$$
\begin{aligned}
\|A(T)-A(\tau)\|_{L^{\infty}\left(M_{r}\right)} & \leq C \sum K^{+}(\tau+i) \\
& \leq C K^{+}(\tau)=C \delta_{1} .
\end{aligned}
$$

If $\delta_{1}$ is small enough that $C \delta_{1}<\epsilon / 2$, we conclude

$$
\begin{aligned}
\left\|u(A(T))-A_{0}\right\|_{L^{4}\left(M_{r}\right)} & \leq\|u(A(T))-u(A(\tau))\|_{L^{4}\left(M_{r}\right)}+\left\|u(A(\tau))-A_{0}\right\|_{L^{4}\left(M_{2 r / 3}\right)} \\
& \leq C \delta_{1}+\epsilon_{1}<\epsilon .
\end{aligned}
$$

Hence, by Lemma 3.6, the inequality (3.16) continues beyond $t=T$, which is a contradiction.

Therefore $T=\infty$, and the above estimates continue as $t \rightarrow \infty$. Letting $\tau$ increase, (3.23) implies exponential convergence in $L^{\infty}\left(M_{r}\right)$ to a limit $A_{\infty}$, as desired. For the Sobolev bounds, note that (3.22) and (3.23), with $H^{k}$ in place of $L^{\infty}$, give

$$
\left\|A(t)-A_{\infty}\right\|_{H^{k}\left(M_{r}\right)} \leq C \delta_{1} e^{-(t-\tau) / C_{1}}\left(1+\sup _{\tau \leq s}\|A(s)\|_{H^{k-1}\left(M_{r / 2}\right)}^{k}\right) .
$$

Assume the desired bound for $k-1$. Over $M_{r / 2}$, we have

$$
\begin{aligned}
\|A(s)\|_{H^{k-1}}^{k} & \leq\left(\|A(\tau)\|_{H^{k-1}}+\|A(s)-A(\tau)\|_{H^{k-1}}\right)^{k} \\
& \leq\left(\|A(\tau)\|_{H^{k-1}}+C \delta_{1}\left(1+\|A(\tau)\|_{H^{k-2}}^{(k-1) !}\right)\right)^{k} \\
& \leq C\left(1+\|A(\tau)\|_{H^{k-1}}^{k !}\right)
\end{aligned}
$$

for $s \geq \tau$, since $\delta_{1}<\bar{\delta}_{1}$. Substituting into (3.24) gives the $k$ 'th Sobolev bound on $M_{r}$.

Note that Theorem 2.5 and (3.19) also imply that the curvature does not concentrate anywhere on $M$ as $t \rightarrow \infty$. The above estimates, with $\left\{x_{i}\right\}=\emptyset$, imply smooth convergence on all of $M$.

Corollary 3.8. Assume that $\left\|F^{+}\right\|<\delta$, and there exists an Uhlenbeck limit $\left(E_{\infty}, A_{\infty}\right)$ which is an instanton with $H_{A_{\infty}}^{2+}=0$. Then $E_{\infty}=E$, and the flow converges smoothly to a connection gauge-equivalent to $A_{\infty}$. 
Proof. By assumption, there exist times $t_{i} \rightarrow \infty$ for which (3.14) and (3.15) are satisfied. Since $F_{A_{\infty}}^{+}=0$ and $\left\|F^{+}(t)\right\|_{L^{\infty}(M)}$ is uniformly bounded, (3.13) is also satisfied for $\tau=t_{i}$ sufficiently large. Therefore, smooth convergence $A(t) \rightarrow A(\infty)$ on $M$ follows from Theorem 3.7. The limit, $A(\infty)$, is related to $A_{\infty}$ by a bundle map $u=\lim u_{i}$ defined on $M_{0}$. Both connections are smooth, and $u \in L^{\infty}(M)$ by definition. Bootstrapping via (1.5) shows that $u$ is itself smooth over $\left\{x_{i}\right\}$, and $u(A(\infty))=A_{\infty}$ on $M$.

Remark 3.9. The results of this section are strongly analogous to those of Topping [31] on harmonic map flow between spheres. He concludes uniqueness of the positions of the bubbles, whereas we show that they do not form. See [34] for further discussion of the contrast between harmonic map and Yang-Mills flow, also explored by Grotowski and Shatah [13] in the equivariant setting.

\section{Global Behavior near the minimum energy}

In this final section, we derive several consequences of the above results for the global behavior of the Yang-Mills flow at low self-dual energy. The assumptions and notation remain as in Section 3 .

We begin with a trivial gauge-fixing lemma which is convenient for controlling the flow at short time, for non-simply-connected $M$. The proof expresses the fact that two flat connections which are $L^{p}$ close, $p \geq 1$, are close modulo gauge in any norm.

Lemma 4.1. Let $0<r<R_{0}, 1 \leq p \leq q \leq \infty$. Assume that $A_{1}, \bar{A}_{1}, A_{2}, \bar{A}_{2}$, are smooth connections on $E$ over $\Omega \subset M$, with $\bar{A}_{1}, \bar{A}_{2}$ flat. There exists $\bar{\epsilon}>0$ as follows.

If, for $\epsilon<\bar{\epsilon}$, both

$$
\left\|A_{1}-A_{2}\right\|_{L^{p}(\Omega)} \leq \epsilon
$$

and

$$
\left\|A_{1}-\bar{A}_{1}\right\|_{L^{q}(\Omega)}+\left\|A_{2}-\bar{A}_{2}\right\|_{L^{q}(\Omega)} \leq \epsilon
$$

then there exists a gauge transformation $u$ over $\Omega_{r}$ such that

$$
\left\|u\left(A_{1}\right)-A_{2}\right\|_{L^{q}\left(\Omega_{r}\right)} \leq Q_{4.1}^{\epsilon} .
$$

The constant and $\bar{\epsilon}$ depend on $\Omega, r, p, q$, and the structure group of $E$.

Proof. If $\Omega$ is simply-connected, then there exist gauge transformations $u_{1}, u_{2}$ such that $u_{1}\left(\bar{A}_{1}\right)=u_{2}\left(\bar{A}_{2}\right)=0$. The triangle inequality, applied to (4.2), implies

$$
\left\|u_{1}\left(A_{1}\right)-u_{2}\left(A_{2}\right)\right\|_{L^{q}(\Omega)} \leq 2 \epsilon .
$$

Choosing $u=u_{2}^{-1} u_{1}$, we obtain the desired result.

In general, (4.1), (4.2), and the triangle inequality imply that $\left\|\bar{A}_{1}-\bar{A}_{2}\right\|_{L^{p}(\Omega)} \leq C \epsilon$. Fix a finite cover $\left\{B^{a}\right\}_{a=1}^{N}$ of $\Omega_{r}$, consisting of geodesic balls of radius $r / 2$ centered in $\Omega_{r}$. Assume, without loss of generality, that in the local gauge on each $B^{a}$, the connection matrix $\bar{A}_{2}^{a} \equiv 0$. Then, since $p \geq 1$ and $\left\|\bar{A}_{1}^{a}\right\|_{L^{p}\left(B^{a}\right)} \leq C \epsilon$ for all $a$, we may choose points $x^{a} \in B^{a}$ with the 
following property. For the minimizing geodesic $\gamma$ between $x^{a}$ and $x^{b}$ inside $B^{a} \cup B^{b}$, there holds

$$
\int_{\gamma \cap B^{a}}\left|\bar{A}_{1}^{a}(\gamma(t)) \| \gamma^{\prime}\right| d t \leq C \epsilon
$$

and similarly for $\bar{A}_{1}^{b}$ over $B^{b}$. Now let $u^{a}$ be the radial gauge for $\bar{A}_{1}^{a}$ on $B^{a}$ centered at $x^{a}$, with $u^{a}\left(x^{a}\right)=1$, and put

$$
w^{a b}=\left.u^{b}\right|_{B^{a}}\left(u^{a}\right)^{-1}
$$

Then by (4.3), for $\epsilon \leq \bar{\epsilon}$, we have

$$
\left|\log w^{a b}\right|+\left|d w^{a b}\right| \leq C \epsilon .
$$

over $B^{a} \cap B^{b}$. In fact, the $w^{a b}$ are initially constant; we then modify the local frames $u^{a}$ to obtain a frame $u$ over $\Omega_{r}$. Write

$$
U^{c}=\bigcup_{a=1}^{c} B^{a} .
$$

As a base case, on $U^{1}=B^{1}$, let $u=u^{1}$, so that $u\left(\bar{A}_{1}\right) \equiv 0$. Assume, by way of induction, that (4.4) holds for all $a, b$; and moreover that for $a, b<c, u^{a}$ and $u^{b}$ agree on $B^{a} \cap B^{b}$ and hence define a frame $u$ on $U^{c-1}$ such that

$$
\left\|u\left(\bar{A}_{1}\right)-\bar{A}_{2}\right\|_{L^{\infty}\left(U^{c-1}\right)} \leq C \epsilon
$$

Since $B^{c} \cap U^{c-1}$ has finitely many connected components, one can easily write down a gauge $w$ on $B^{c}$ with $w=w^{a c}$ on $B^{a} \cap B^{c}$ for all $a<c$, and $|d w|<C \epsilon$. We then modify

$$
u^{c} \rightarrow w \cdot u^{c} .
$$

The result agrees with $u$ on the overlap, and again satisfies (4.4). Since the balls $B^{a}$ are fixed and of finite number, for $\epsilon<\bar{\epsilon}$ the induction hypotheses (4.4), (4.5) will be satisfied appropriately.

We have now constructed a gauge transformation $u$ such that $\left\|u\left(\bar{A}_{1}\right)-\bar{A}_{2}\right\|_{L^{\infty}\left(\Omega_{r}\right)} \leq C \epsilon$. Over $\Omega_{r}$, the triangle inequality gives

$$
\left\|u\left(A_{1}\right)-A_{2}\right\|_{L^{q}} \leq\left\|u\left(A_{1}\right)-u\left(\bar{A}_{1}\right)\right\|_{L^{q}}+\left\|u\left(\bar{A}_{1}\right)-\bar{A}_{2}\right\|_{L^{q}}+\left\|\bar{A}_{2}-A_{2}\right\|_{L^{q}} \leq C \epsilon
$$

as desired.

Theorem 4.2. (Theorem 1.1 of Taubes [30], parabolic version.) Let $(\bar{E}, \bar{A})$ be a flat bundle on $M$ with $H_{\bar{A}}^{2+}=0$, and let $r$ and $\epsilon$ be as in Lemma 3.6. For any $p>1, K^{+}>0$, and points $x_{1}, \ldots, x_{m} \in M$, there exist $\delta_{1}, \epsilon_{1}>0$ as follows. If $A(0)$ is a connection on $E$ with

$$
\begin{gathered}
\left\|F_{A(0)}^{+}\right\|_{L^{2 p}(M)} \leq K^{+} \\
\left\|F_{A(0)}^{+}\right\|_{L^{2}(M)} \leq \delta_{1} \\
\|u(A(0))-\bar{A}\|_{H^{1}\left(M_{r / 2}\right)} \leq \epsilon_{1}
\end{gathered}
$$


then the flow with initial data $A(0)$ converges smoothly as $t \rightarrow \infty$ to an instanton near $\bar{A}$ in $L^{4}\left(M_{r}\right)$ modulo gauge 10

Proof. Assuming $C_{1.16} \epsilon_{1}<\epsilon$, (4.6) and the local Sobolev inequality (1.16) imply both a Poincaré estimate $(3.12)$ for $A(0)$, and an initial energy bound

$$
\|F(0)\|_{L^{2}\left(M_{3 r / 2}\right)}<C \epsilon_{1} .
$$

To control the curvature for a short time, we apply the estimates of Section 2.1, with $u(t)=\left|F^{+}(t)\right|^{2}$. The proof of Proposition 2.1, as given for the $L^{2}$ norm, can with heavier notation be adapted to the $L^{p}$ norm, for any $p>1$, to give a uniform bound

$$
\|u(t)\|_{L^{p}} \leq K^{+}+C .
$$

Proposition 2.3, with the explicit constant, then implies $\|u(t)\|_{L^{\infty}} \leq C\left(1+t^{-2 / p}\right)$, i.e.

$$
\left\|F^{+}(t)\right\|_{L^{\infty}} \leq C\left(1+t^{-1 / p}\right) .
$$

Since $p>1$, the function $\left\|F^{+}(t)\right\|_{L^{\infty}}$ is $L^{1}$, and there exists $0<\tau<1$ such that

$$
\int_{0}^{\tau}\left\|F^{+}(t)\right\|_{L^{\infty}(M)} d t<\epsilon_{1} .
$$

Theorem 2.5 then implies

$$
\|F(t)\|_{L^{2}\left(M_{r}\right)}<C \epsilon_{1}
$$

for $0 \leq t \leq \tau$.

Assume first that $M$ is simply-connected. From Proposition 3.2 and the energy inequality, the curvature at time $\tau$ on $M_{3 r / 2}$ is bounded by $C\left(\delta_{1}+\epsilon_{1}\right)$, as are all its derivatives. We therefore have the curvature-dependent bounds sufficient for the Coulomb gauge patching argument of [10], §4.4.2 I1] In particular, Proposition 4.4.10 of [10] states that for $\delta_{1}$ sufficiently small, there exists a gauge transformation $u$ on $M_{r}$ with

$$
\|u(A(\tau))-\bar{A}\|_{L^{4}\left(M_{r}\right)}<C \epsilon_{1} .
$$

The existence of $\delta_{1}>0$ as required now follows from Theorem 3.7 .

If $M$ is not simply-connected, we argue as follows. Let $\pi: \tilde{M} \rightarrow M$ be the universal cover, and choose a strongly simply-connected domain $\Omega \subset \tilde{M}$ covering $M_{r}$, which is a finite union of preimages of $B^{a} \subset M_{r / 2}$, with $B^{a} \cap B^{b}$ connected12] By [10], Prop. 4.4.10, as before we may choose a gauge $v$ on $\Omega_{r / 2}$ such that

$$
\left\|v\left(\pi^{*} A(\tau)\right)-\pi^{*} \bar{A}\right\|_{L^{4}\left(\Omega_{r / 2}\right)}<C \epsilon_{1} .
$$

\footnotetext{
${ }^{10}$ In fact it will be close over $M_{r}$ in any Sobolev norm for $\delta_{1}+\epsilon_{1}$ sufficiently small, as remarked in [10] $\S 4.4 .2$.

${ }^{11}$ Proposition 3.2 exactly replaces [10, Theorem 2.3.8, which gives curvature-dependent bounds from the ASD equation.

${ }^{12}$ This can be done for instance by lifting the $B^{a}$ to $\tilde{M}$ using a set of paths which form a spanning tree for the incidence graph of $\left\{B^{a}\right\}$.
} 
If this is done using preimages of Coulomb gauges on the $B^{a}$, then $v^{-1}\left(\pi^{*} \bar{A}\right)$ descends to a flat connection on $E$ over $M_{r / 2}$. Calling this connection $\bar{A}_{1}$, we obtain

$$
\left\|A(\tau)-\bar{A}_{1}\right\|_{L^{4}\left(M_{r / 2}\right)} \leq C \epsilon_{1} .
$$

Note also, directly from the flow equation and the energy inequality, that

$$
\|A(\tau)-A(0)\|_{L^{2}(M)} \leq \tau^{1 / 2}\left(\int_{0}^{\tau}\left\|D^{*} F\right\|_{L^{2}(M)}^{2} d t\right)^{1 / 2} \leq \delta_{1} .
$$

In view of (4.6), (4.8), and (4.9), we may apply Lemma 4.1 with $p=2$ and $q=4$ to $A(0), A(\tau)$, and the two flat connections $\bar{A}, \bar{A}_{1}$. We conclude that there in fact exists a gauge transformation $u$ on $M_{r}$ with

$$
\|u(A(\tau))-A(0)\|_{L^{4}\left(M_{r}\right)} \leq Q_{4.1}\left(\delta_{1}+\epsilon_{1}\right) .
$$

The desired result follows again from Theorem 3.7 .

Theorem 4.3. Assume $E$ has structure group $S U(2)$ with $\kappa(E)=1, H^{2+}(M)=0$, and $\pi_{1}(M)$ has no nontrivial representations in $S U(2)$. There exists $\delta_{1}>0$ such that if $\left\|F^{+}(0)\right\|<$ $\delta_{1}$, then no bubbling occurs as $\rightarrow \infty$. If an Uhlenbeck limit $A_{\infty}$ is an instanton with $H_{A_{\infty}}^{2+}=0$, then it is unique, and the flow converges exponentially.

Proof. Assume, by way of contradiction, that bubbling occurs on a sequence $t_{i} \rightarrow \infty$. The blowup limits constructed by Schlatter [22] at a presumed singularity, as well as the Uhlenbeck limit, preserve the structure group. Due to the $L^{\infty}$ bound on $F^{+}$, the blowup limit at a bubble must be anti-self-dual. Since it is a nontrivial $S U(2)$ instanton on $S^{4}$ with energy less than $4 \pi^{2}+\delta_{1}^{2}$, it must have energy exactly $4 \pi^{2}$.

Let $A_{\infty}$ be the Uhlenbeck limit obtained from Theorem 3.5 on the same sequence $\left\{t_{i}\right\}$, which has total energy less than $\delta_{1}^{2}$. We may apply [10], Prop. 4.4.10, on the universal cover of $M$ as in the preceding proof (with $\left\{x_{i}\right\}=\emptyset$ ). This implies that for $\delta_{1}$ sufficiently small, $A_{\infty}$ must be $L^{4}$-close to a flat connection $\bar{A}$ on $M$, modulo gauge. But, by the assumption on $\pi_{1}(M), \bar{A}$ is equivalent to the product connection on the trivial bundle, and so

$$
H_{\bar{A}}^{2+}=H^{2+}(M)=0
$$

Hence, for $\delta_{1}$ sufficiently small, $A_{\infty}$ satisfies a Poincaré estimate (3.12). By Theorem $\underline{3.5}, A_{\infty}$ is Yang-Mills, hence by (1.11) and (3.12) it must be anti-self-dual. By integrality of $\kappa(E)$ for $S U(2)$-bundles, $A_{\infty}$ is flat, and also equivalent to the product connection. Corollary 3.8 then implies that the flow converges, which is a contradiction.

Therefore any Uhlenbeck limit exists smoothly. The last statement follows again from Corollary 3.8 .

Theorem 4.4. The instantons with $H^{2+}=0$ are asymptotically stable in the $H^{1}$ topology. In other words, given an anti-self-dual connection $A_{1}$ with $H_{A_{1}}^{2+}=0$ and an $H^{1}$ neighborhood $U$ of $A_{1}$, there exists a neighborhood $U_{1} \subset U$ of initial connections for which the limit under the flow will again be an instanton with $H^{2+}=0$, lying in $U$ modulo smooth gauge transformations. 
Proof. Choose the given instanton $A_{1}$ for the required smooth connection $D_{1}=D_{\text {ref }}+A_{1}$ in Struwe's construction-see Section 1.7. Let $\epsilon>0$ be such that the $H^{1}$ neighborhood of $A_{1}$ of size $C_{1.1} \epsilon$ is contained in $U$, and choose $U_{1}$ to be the neighborhood of size $\epsilon$. By Theorem 1.1, the gauge-equivalent flow (1.19) with initial data in $U_{1}$ will remain in $U$ for a time $\tau$. Choosing $U_{1}$ smaller, we also obtain the three conditions (3.13), (3.14), (3.15) with $\delta_{1}$ arbitrarily small. We are then in the situation of Theorem 3.7, which may be applied with $\tau_{0}=\tau$ and $\left\{x_{i}\right\}=\emptyset$.

Corollary 4.5. On any $S U(2)$-bundle $E \rightarrow M$, there exists a nonempty $H^{1}$-open set of initial connections for which the Yang-Mills flow exists for all time and, if $\kappa(E) \geq 0$ and $H^{2+}(M)=0$, converges exponentially.

Proof. Following Freed and Uhlenbeck [11], for any $\delta_{1}$, one can construct smooth pointlike $S U(2)$-connections with $\left\|F^{+}\right\|_{L^{2}}<\delta_{1}$ and $\left\|F^{+}\right\|_{L^{\infty}}<C$ (p. 124). Provided $H^{2+}(M)=0$, for $\delta_{1}$ sufficiently small, Theorem 4.2 yields exponential convergence to an instanton $A_{\infty}$. By Theorem 4.4, convergence holds for initial data in an $H^{1}$-open neighborhood.

Corollary 4.6. There exists a $\mathscr{G}_{E}$-invariant $H^{1}$-open set $N \subset \mathscr{A}_{E}$, such that the flow gives a deformation retraction, with respect to the $H^{k}$ topology on $\mathscr{A}_{E} / \mathscr{G}_{E}$, for $k>>1$, from $N \cap H^{k}$ onto the instantons with $H^{2+}=0$.

Proof. Let $N$ be the union of the neighborhoods obtained in Theorem 4.4, which we may take to be invariant under smooth gauge transformations. By Kozono et. al., Theorem C, for $k$ sufficiently large, the Yang-Mills flow is in fact unique modulo gauge as long as it exists in $H^{k}$, and therefore gives a well-defined map on $N / \mathscr{G}_{E}$ fixing the instantons.

It remains to show that this map is continuous modulo gauge in the $H^{k}$ topology. This follows by combining the parabolic theory of Section 1.7 with the uniform convergence statement of Theorem 3.7. For, two connections in $N$ which are initially $H^{k}$-close, for $k \geq k_{0}$, remain so under the gauge-equivalent flow (1.19). They therefore remain close, modulo gauge, for an arbitrarily long time; but then both are close to their respective limits under the Yang-Mills flow.

To spell this out, first note that since the flow converges smoothly, we always have

$$
\|A(t)\|_{H^{k}}<K
$$

for a constant $K$ depending on $A(0) \in N$. By Section 1.7, there exists $\tau>0$ such that the map

$$
A(t) \rightarrow A(t+\tau)
$$

is continuous in $H^{k}$, modulo gauge, for all solutions satisfying (4.10). Since continuity is a local property and closed under composition, we conclude that for any $0 \leq T<\infty$, the map $A(t) \rightarrow A(t+T)$ is continuous modulo gauge on $N \times[0, \infty)$.

Now, let $A_{1}$ be an instanton with $H_{A_{1}}^{2+}=0$, and fix a neighborhood $U \ni A_{1}$. By Theorem 2.5, there exists a sufficiently small neighborhood $V \subset U$ with the property that if $A(t)$ satisfies

$$
A(\tau-1), A(\tau) \in V
$$


for any $\tau \geq 1$, then

$$
\lim _{t \rightarrow \infty} A(t) \in U
$$

To show continuity at infinite time, let $A^{\prime}(t)$ be a solution with $A^{\prime}(0) \in N$ and $\lim _{t \rightarrow \infty} A^{\prime}(t)=$ $A_{1}$. Choose $\tau$ such that $A^{\prime}(t)$ satisfies (4.11). Then by continuity on $[0, \tau]$, for $A^{\prime \prime}(0)$ sufficiently close to $A^{\prime}(0), A^{\prime \prime}(t)$ will also satisfy (4.11) after changing gauge. Therefore $A^{\prime \prime}(t)$ also satisfies (4.12), as desired.

Acknowledgements. These results formed part of the author's PhD thesis [34] at Columbia University, and he thanks his advisor, Panagiota Daskalopoulos, for vital direction and support. Thanks also to Richard Hamilton for noticing a simplification, to Michael Struwe for an encouraging discussion, and to D. H. Phong for initially suggesting the problem.

\section{REFERENCES}

[1] M. F. Atiyah and R. Bott. The Yang-Mills equations over Riemann surfaces. Philosophical Transactions of the Royal Society of London. Series A, Mathematical and Physical Sciences (1983), 523-615.

[2] J.-P. Bourguignon, H. B. Lawson. Stability and isolation phenomena for Yang-Mills fields. Comm. Math. Phys. 79 (1981), no. 2, 189230.

[3] K. C. Chang, W. Y. Ding, and R. Ye. Finite-time blowup of harmonic maps from surfaces. J. Diff. Geom. 36 (1992), 507-515.

[4] Y. Chen. and C.-L. Shen. Monotonicity formula and small action regularity for Yang-Mills flows in higher dimensions. Calc. Var. 2 (1994), 389-403.

[5] G. Daskalopoulos. The topology of the space of stable bundles on a compact Riemann surface. J. Differential Geom. 36 (1992), no. 3, 699-746.

[6] G. Daskalopoulos and R. Wentworth. Convergence properties of the Yang-Mills flow on Kahler surfaces. J. Reine Angew. Math. 575 (2004), 69-99.

[7] - . On the blow-up set of the Yang-Mills flow on Kahler surfaces. Math. Z. 256 (2007), no. 2, 301-310.

[8] S. K. Donaldson. An application of gauge theory to four dimensional topology. J. Differential Geom. 18 (1983), 279-315.

[9] - Anti self-dual Yang-Mills connections over complex algebraic surfaces and stable vector bundles. Proceedings of the London Mathematical Society 50.1 (1985), 1-26.

[10] S. K. Donaldson and P. B. Kronheimer. The Geometry of Four-Manifolds. Oxford University Press (1990).

[11] D. Freed and K. Uhlenbeck. Instantons and Four-Manifolds. MSRI Research Publications I (1984).

[12] D. Gilbarg, and N. S. Trudinger. Elliptic Partial Differential Equations of Second Order. Springer (1983).

[13] J. Grotowski and J. Shatah. Geometric evolution equations in critical dimensions. Calc. Var. 30 (2007), 499-512.

[14] R. S. Hamilton. Monotonicity formulas for parabolic flows on manifolds. Comm. Anal. Geom 1.1 (1993), $127-137$.

[15] M. C. Hong and G. Tian. Asymptotical behavior of the Yang-Mills flow and singular Yang-Mills connections. Math. Ann. 330 (2004), 441-472.

[16] H. Kozono, Y. Maeda, H. Naito. Global solution for the Yang-Mills gradient flow on 4-manifolds. Nagoya Math. J. 139 (1995), 93128.

[17] H. B. Lawson. The Theory of Gauge Fields in Four Dimensions. Regional Conference Series in Mathematics, Number 58, AMS (1985).

[18] P. Li. Geometric Analysis. Cambridge University Press (2012).

[19] J. L. Lions, and E. Magenes. Non-Homogeneous Boundary Value Problems and Applications. Springer (1973). 
[20] H. Naito. Finite time blowing-up for Yang-Mills gradient flow in higher dimensions. Hokkaido Math. J. 23 (1994), no. 3, 451-464.

[21] J. Rade. On the Yang-Mills heat equation in two and three dimensions. J. Reine Angew. Math. 120 (1998), 117-128.

[22] A. E. Schlatter. Long-time behavior of the Yang-Mills flow in four dimensions. Ann. Global Anal. Geom. 15 (1997), no. 1, 1-25.

[23] —. Global existence of the Yang-Mills flow in four dimensions. J. Reine Angew. Math. 479 (1996), 133-148.

[24] R. Schoen. Analytic aspects of the harmonic map problem. Seminar on nonlinear partial differential equations, Springer (1984), 321-358.

[25] A. E. Schlatter, M. Struwe and A. S. Tahvildar-Zadeh. Global existence of the equivariant Yang-Mills heat flow in four space dimensions. Am. J. Math. 120 (1998), 117-128.

[26] S. Sedlacek. The Yang-Mills functional over four-manifolds. Comm. Math. Phys. 86 (1982), 515-527.

[27] Y. T. Siu. Lectures on Hermitian-Einstein Metrics for Stable Bundles and Kahler-Einstein Metrics. Birkhauser, 1986.

[28] M. Struwe. On the evolution of harmonic mappings of Riemann surfaces. Comment. Math. Helv. 60 (1985), no. 4, 558-581.

[29] —. The Yang-Mills flow in four dimensions. Calc. Var. 2 (1994), 123-150.

[30] C. H. Taubes. Self-dual Yang-Mills connections on non-self-dual 4-manifolds. J. Diff. Geom. 17.1 (1982), 139-170.

[31] P. M. Topping. Rigidity in the harmonic map heat flow. J. Diff. Geom. 45.3 (1997), 593-610.

[32] K. Uhlenbeck. Connections with $L^{p}$ bounds on curvature, Comm. Math. Phys. 83 (1982), 31-42.

[33] K. Uhlenbeck. Removable singularities in Yang-Mills fields, Comm. Math. Phys. 83 (1982), 11-30.

[34] A. Waldron. Self-duality and singularities in the Yang-Mills flow. Ph.D thesis, Columbia University, 2014.

[35] B. Weinkove. Singularity formation in the Yang-Mills flow. Calc. Var. 19 (2004), 221-220. 